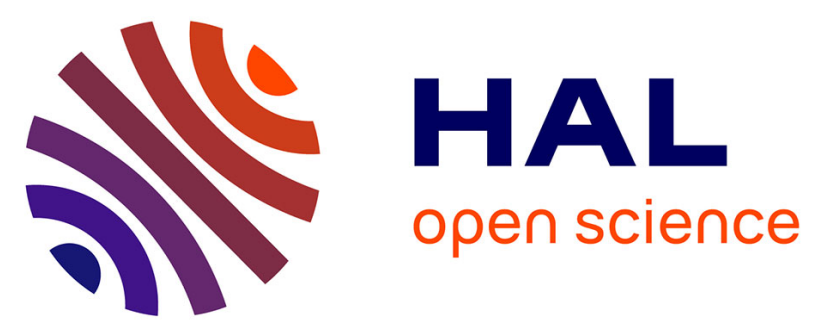

\title{
Identification and assessment of ecotoxicological hazards attributable to pollutants in urban wet weather discharges
}

Antoine Gosset, Claude Durrieu, Frédéric Orias, Rémy Bayard, Yves Perrodin

\section{- To cite this version:}

Antoine Gosset, Claude Durrieu, Frédéric Orias, Rémy Bayard, Yves Perrodin. Identification and assessment of ecotoxicological hazards attributable to pollutants in urban wet weather discharges. Environ. Sci.: Processes Impacts, 2017, 19, pp.1150-1168. 10.1039/C7EM00159B . hal-01567078

\section{HAL Id: hal-01567078 \\ https://hal.science/hal-01567078}

Submitted on 21 Jul 2017

HAL is a multi-disciplinary open access archive for the deposit and dissemination of scientific research documents, whether they are published or not. The documents may come from teaching and research institutions in France or abroad, or from public or private research centers.
L'archive ouverte pluridisciplinaire HAL, est destinée au dépôt et à la diffusion de documents scientifiques de niveau recherche, publiés ou non, émanant des établissements d'enseignement et de recherche français ou étrangers, des laboratoires publics ou privés. 


\section{This paper is available at pubs.rsc.org}

1 Identification and assessment of ecotoxicological hazards attributable to pollutants in 2 urban wet weather discharges

3

Antoine Gosset ${ }^{1,2 *}$, Claude Durrieu ${ }^{1}$, Frédéric Orias ${ }^{1}$, Rémy Bayard $^{2}$, Yves Perrodin ${ }^{1}$

${ }^{1}$ Université de Lyon, ENTPE, CNRS, UMR 5023 LEHNA, 3 Rue Maurice Audin, 69518 Vaulx-en-

Velin, France

${ }^{2}$ Université de Lyon, INSA-Lyon, Laboratoire DEEP, Bât. S. Carnot, 9 rue de la Physique, F-69621

Villeurbanne, France

*corresponding author. Université de Lyon, ENTPE, CNRS, UMR 5023 LEHNA, 3 Rue Maurice Audin, 69518 Vaulx-en-Velin, France. Tel.: +33 4720472 90; Fax: +33 472047743.

E-mail address: antoine.gosset@entpe.fr

\section{$\underline{\text { Abstract }}$}

The phenomenon of urbanization leads to considerable pressure on urban/suburban aquatic ecosystems. Urban Wet Weather Discharges (UWWDs) during rainfall events are a major source of pollutants leached onto and into urban surfaces and sewers, which in turn affect aquatic ecosystems. The aim of this study is to assess the ecotoxicity of the different compounds identified in UWWDs and identify the hazard represented by each of them. To this end, hazard quotients (HQ) were calculated for each compound detected in UWWDs, based on their Predicted No Effect Concentration (PNEC) values and their maximum concentration ( $\mathrm{MEC}_{\max }$ ) found in the dissolved part of UWWDs. For the 207 compounds identified in UWWDs, sufficient data existed for 165 of them to calculate their PNEC. The ecotoxicity of these compounds is greatly variable. Pesticides represent a high proportion of the wide variety of hazardous compounds whose HQ were calculated (a total of 94 HQ), and they are among the most hazardous pollutants $(\mathrm{HQ}>1000)$ transported by stormwater. On the contrary, the hazard of Combined Sewer Overflows (CSO) is mainly linked to heavy metals and pharmaceutical compounds. Consequently, the monitoring of these pollutants is a priority in the future. Lastly, it is to bear in mind that the hazard level of certain pollutants could be underestimated, due to their adsorption on particles, leading their low presence in the dissolved phase of UWWDs. So, an in-depth study of these pollutants will be required to clarify their effects on aquatic organisms.

How to cite this paper: Gosset, A., Durrieu, C., Orias, F., Bayard, R., Perrodin, Y. (2017) Identification and assessment of ecotoxicological hazards attributable to pollutants in urban wet weather discharges. Environmental Science: Processes \& Impacts DOI: 10.1039/C7EM00159B 


\section{This paper is available at pubs.rsc.org}

$\underline{\text { Introduction }}$

Urban Wet Weather Discharges (UWWDs) are complex and variable polluted matrices, notably due to the different types of catchments leached by the rain, and the variable flows for different events. ${ }^{1}$ Many micropollutants have been detected in these effluents as a function of population density and anthropogenic activities. Among them, we can cite here: (i) European priority substances such as Polycyclic Aromatic Hydrocarbons (PAHs), ${ }^{2-5}$ diethylexylphtalate, ${ }^{4,6}$ 4-nonylphenol, ${ }^{4,6}$ bisphenol A and alkylphenolethoxylate ${ }^{7}$, (ii) other micropollutants like heavy metals (copper, lead, zinc, etc.), polychlorobiphenyls (PCBs), pesticides, phthalates ${ }^{3,5,8,9,10}$, and (iii) pharmaceutical residues (PC) in the particular case of the Combined Sewer Overflows $(\mathrm{CS} 0)^{11,12}$. These micropollutants have also already been detected in receiving river water having received these discharges. ${ }^{13,14}$ Many of them are potentially hazardous for aquatic organisms. That is why UWWDs have toxic effects on aquatic species, ${ }^{15,16,17}$ and can thus modify biological communities. ${ }^{15,18,19,20,21}$ Treatment to diminish UWWD pollutants through abatement are nonexistent or very limited depending on municipal sewer systems, and raw UWWD are often release into (sub)urban aquatic ecosystems. It is therefore necessary to optimize their management. This optimization can concern: (i) a better monitoring of toxic pollutants and their effects, including continuous monitoring with biosensors; $;^{22,23}$ (ii) a specific treatment of pollutants; ${ }^{24}$ and (iii) the suppression or the substitution at source of the toxic products at the origin of these pollutants (in particular, certain detergents, disinfectants and pharmaceuticals) by less toxic but equally efficient products. For that, it is necessary to identify and prioritize the hazardous pollutants present in UWWDs. To establish the list of the compounds most concerned by the ecotoxicological hazard represented by a specific urban effluent, many authors have proposed to formulate and apply a hazard quotient based on the ratio "Concentration of the selected pollutant in filtered polluted matrices"/"Predicted No Effect Concentration (PNEC) of the molecules selected". ${ }^{25,26,}$ This quotient is used to distinguish the hazardousness of the different compounds and families of compounds found in UWWDs. Orias and Perrodin ${ }^{25}$ proposed comparing the maximum Measured Effluent Concentrations (MECmax) measured in hospital effluents to their PNEC value, which is the method used in the current study. This method was also applied by many other authors for contaminated matrices such as rivers polluted by raw or treated municipal wastewaters polluted by various pharmaceuticals,${ }^{26}$ pesticides, ${ }^{27}$ or

How to cite this paper: Gosset, A., Durrieu, C., Orias, F., Bayard, R., Perrodin, Y. (2017) Identification and assessment of ecotoxicological hazards attributable to pollutants in urban wet weather discharges. Environmental Science: Processes \& Impacts DOI: 10.1039/C7EM00159B 


\section{This paper is available at pubs.rsc.org}

lakes by antibiotics. ${ }^{28}$ The choice of using the maximum value of MEC (MECmax) is to be place us in the worst (extreme conditions) case scenario ${ }^{25,27}$ and in the context of the precautionary principle. This method was applied here for the first time to the UWWDs, translating its originality.

Consequently, the main objectives of our study were to: (i) conduct a review of the ecotoxicity data available for the different compounds commonly detected in UWWDs; (ii) assess the concentrations of these pollutants in UWWDs performing a literature review of UWWDs pollutant compositions; and (iii) calculate the potential hazard of these pollutants for aquatic ecosystems. Several studies have already proposed to identify and prioritize hazardous pollutants in UWWDs. ${ }^{29-32}$ Nevertheless, they generally considered only the stormwater (no studies were reported for the CSO). Moreover, these pollutant hazard assessments were based only on the intrinsic properties of the pollutants (bioaccumulation, biodegradability, physicochemistry, ecotoxicity) and did not take into account the concentrations found in situ.

In this article, we present the PNEC values inventoried for all the pollutants identified in UWWDs, and the MECmax found in these discharges. Lastly, we calculated the HQ for each pollutant found in UWWDs. We also chose to focus on combined and separate stormwater systems (named here "CSO" and "stormwater" systems) due to the very different sources and types of pollutants they contain.

\section{Methodology}

All the pollutants used and analyzed (on dissolved and particles phases of UWWDs) in this study were classified into families of chemical compounds, as detailed in Table 1. Scientific research papers for UWWD pollutants identification were obtained using databases such as the Web of Knowledge, Science Direct and Google Scholar, using key search terms (e.g. "stormwater", "CSO", "UWWD", "pollutants", “chemicals"). An identified paper's bibliography and citations were also checked to find other suitable studies. Finally, papers used in this study were restricted to peer-reviewed research articles published between 1995 and 2016. Other scientific productions (e.g. conference papers, non-peer reviewed procedures) were not retained for this study.

\subsection{Obtaining and determining the PNEC values of micropollutants in UWWDs}

How to cite this paper: Gosset, A., Durrieu, C., Orias, F., Bayard, R., Perrodin, Y. (2017) Identification and assessment of ecotoxicological hazards attributable to pollutants in urban wet weather discharges. Environmental Science: Processes \& Impacts DOI: 10.1039/C7EM00159B 


\section{This paper is available at pubs.rsc.org}

To define the hazard of the different pollutants identified in UWWD physico-chemical studies, the first step of our method was to determine their ecotoxicity by seeking or calculating their PNEC. PNEC values were found/calculated for all pollutants sought in physico-chemical studies (on particle and dissolved phases). In this study, the PNEC values were obtained for all the pollutants using the following approach: (i) When they were available, PNEC values were obtained from free European databases $^{33,34}$ (INERIS and AGRITOX) containing ecotoxicological data from international scientific literature, (ii) When values were not available in these databases, PNEC values were calculated using experimental ecotoxicological data found in the EPA ECOTOX database ${ }^{35}$, following the method proposed by Orias et Perrodin, ${ }^{36}$ and in agreement with the guidelines proposed by the EU (European Union) in the Technical Guidance Document (TGD) ${ }^{37}$ (see Table 2). This method is the following: for each compound, bibliographical research using the EPA ECOTOX database allowed identifying all the bioassays performed, the trophic level concerned (decomposers, primary producers, primary consumers and secondary consumers) and the chronicity (or not) of the bioassay (e.g. reproduction). Using all the ecotoxicity data, an Extrapolation Factor (EF), calculated following the criteria presented in Table S1, was applied to the lowest toxicity values to establish the PNEC of the compound concerned. No differentiation was done between the ecotoxicity data used (e.g. $\left.\mathrm{EC}_{50}, \mathrm{EC}_{10}\right)$ for the $\mathrm{EF}$ and PNEC calculation. However, several minor modifications in comparison to TGD were made: ecotoxicity data on fungi were considered as data on decomposers while data on periphyton were considered to originate from chronic bioassays on decomposers. In the case where only acute ecotoxicity data were available, but not for all trophic levels, PNEC values were calculated with the ECOSAR model, by applying an EF to the lowest ecotoxicological value. In all cases, no PNEC values were calculated if at least one acute ecotoxicity value was available for each trophic level. The chronic algae and macrophyte bioassays did not permit lowering the EF when they were the only ecotoxicological data available. Finally, the EF was not reduced when rotifer (Brachionus calyciflorus, noted "Bc") bioassays were the only tests performed on the trophic level of secondary consumers. ${ }^{37}$ Finally, when no PNEC and ecotoxicity data were available in databases and literature, PNEC values were estimated with modelled ecotoxicological data obtained using the ECOSAR method. ${ }^{38}$

How to cite this paper: Gosset, A., Durrieu, C., Orias, F., Bayard, R., Perrodin, Y. (2017) Identification and assessment of ecotoxicological hazards attributable to pollutants in urban wet weather discharges. Environmental Science: Processes \& Impacts DOI: 10.1039/C7EM00159B 


\section{This paper is available at pubs.rsc.org}

It is important to point out that the authors of several studies did not indicate the speciation or

the isomer of the compounds measured. This is the case of chromium, for which we chose to keep the two speciation forms (chromium III and chromium VI), as it has been reported that the annual concentrations of these two chromium species in rainwater samples are similar. ${ }^{39}$ In the case of dichloroethane, we chose to keep the two isomers (1-1 and 1-2-dichloroethane), while the hazard quotient was calculated twice for this pollutant, using two PNECs.

\subsection{Obtaining micropollutant MECmax}

The second step for UWWD pollutant hazard assessment was the $\mathrm{MEC}_{\max }$ determination for each of them. As described in Orias and Perrodin ${ }^{25}$, two different approaches can be used to obtain the concentration of micropollutants in UWWDs: (i) a theoretical method based on the calculation of PEC (Predicted Effluent Concentration) with the design of a scenario, and (ii) an experimental approach based on analyses of the pollutants in the dissolved phase of the UWWDs. The second method (MECmax determination) is more realistic, because it is based on real measured concentrations in UWWDs, contrary to the calculation of PEC values presenting a high level of uncertainty due to the parameters used (e.g. product consumption). Therefore, this option was selected although not all the compounds were analyzed in the discharges.

In this study, we will discuss the different choices made to select concentration data (see section 3.5). Firstly, we considered studies providing compound concentration values only in the soluble phase $(<1.2 \mu \mathrm{m})$. Indeed, ecotoxicity assays performed to calculate PNEC values for the different pollutants have relied only on pure liquid solutions. Moreover, it is impossible to evaluate the bioavailability of the contaminants in the particulate phase for aquatic organisms. Additionally, taking into account the concentrations on particles could lead to overestimating the hazard (see section 3.5). Regarding the formulation of the Hazard Quotient (HQ), we also chose to separate concentration values for separate and combined sewer systems, thus we calculated the hazard for both cases. The hazards of these two systems differ, as the source and type of pollutants (i.e. the presence of pharmaceuticals in discharges from combined sewer overflows) are also different.

How to cite this paper: Gosset, A., Durrieu, C., Orias, F., Bayard, R., Perrodin, Y. (2017) Identification and assessment of ecotoxicological hazards attributable to pollutants in urban wet weather discharges. Environmental Science: Processes \& Impacts DOI: 10.1039/C7EM00159B 


\section{This paper is available at pubs.rsc.org}

For each compound sought in the dissolved part of the stormwater/CSO samples, the MEC of the events observed was reported for each study. Finally, the highest effluent concentration ever measured in UWWDs (MEC ${ }_{\max }$ ) was determined by seeking the highest MEC reported previously, with the aim to be situated in the worst-case scenario, as explained in the introduction. In further research, it would be interesting to make the same work with the median and the lowest values, but that will be limited by the fact that for some compounds, only one concentration value was available in scientific literature.

\subsection{Calculation of the hazard quotients}

Finally, for each compound detected in discharges (section 2.2) with an available PNEC value (section 2.1), we calculated an hazard quotient using the equation (1):

(1) $\mathrm{HQ}=$ MECmax./PNEC

With:

MECmax: the highest effluent concentration ever measured in UWWDs in $\mu \mathrm{g} . \mathrm{L}^{-1}$;

PNEC: Predicted No Effect Concentration in $\mu \mathrm{g} \mathrm{L}^{-1}$.

It was considered that compounds with an HQ up to 1 are potentially hazardous for the environment, and that compounds with the highest HQ are the most hazardous for aquatic ecosystems. On the contrary, compounds with an HQ lower than 1 were considered slightly hazardous.

In this study, 207 compounds were identified in UWWDs. We found or calculated/modelled a PNEC value representing the ecotoxicity of 165 of them. By seeking the dissolved proportion analyzed in UWWDs, we were finally able to determine a MECmax for only 61 compounds in "stormwater" and 49 in "CSO". Consequently, we calculated 94 hazard quotients using equation (1) to prioritize their hazardousness for freshwater ecosystems.

3. Results and discussion

3.1 Pollutant classification and available data.

How to cite this paper: Gosset, A., Durrieu, C., Orias, F., Bayard, R., Perrodin, Y. (2017) Identification and assessment of ecotoxicological hazards attributable to pollutants in urban wet weather discharges. Environmental Science: Processes \& Impacts DOI: 10.1039/C7EM00159B 


\section{This paper is available at pubs.rsc.org}

Pollutants analyzed in UWWDs were classified as shown in Table 1. All the PNECs, concentrations and hazard data were analyzed as per the pollutant ranking presented in Table 1.

Table 1: Families of compounds considered in this study and colour codes associated in the various figures.

\begin{tabular}{|c|c|c|c|}
\hline Abbreviation & Family of pollutants & \multicolumn{2}{|c|}{ Figure colour code } \\
\hline ALK & Alkylphenols & & \\
FR & Flame Retardants & & \\
M & Metals & & \\
O & Others & & \\
ORG & Organotins & \\
P & Pesticides & \\
PAH & Polycyclic Aromatic Hydrocarbons and derivatives & \\
PBDE & Polybrominated Diphenyl Ethers & \\
PC & Pharmaceutical Compounds & \\
PCB & Polychlorobiphenyls & \\
PFAA & Perfluoroalkyl Acids & \\
PHT & Phtalates & \\
VOC & Volatile Organic Compounds & \\
\cline { 2 - 5 } & & & \\
\hline
\end{tabular}

\subsection{Ecotoxicity of the pollutants sought in UWWDs}

Of the 207 compounds sought in the UWWD samples (total, dissolved and/or particle phases),

161 were obtained by calculation using data from the European database and the international literature.

In addition, we formulated ourselves 52 new PNEC values based on experimental data and 4 were modelled using ECOSAR model, as detailed in Table 2. Finally, we listed a total of 165 PNEC values. Figure 1 presents the distribution of available PNEC values for each pollutant family. This figure shows that 5 families present a large number of molecules for which PNEC data are not available: PAH, PC, ALK, O and PCB. This lack of data poses a problem for estimating UWWD compound hazards.

How to cite this paper: Gosset, A., Durrieu, C., Orias, F., Bayard, R., Perrodin, Y. (2017) Identification and assessment of ecotoxicological hazards attributable to pollutants in urban wet weather discharges. Environmental Science: Processes \& Impacts DOI: 10.1039/C7EM00159B 


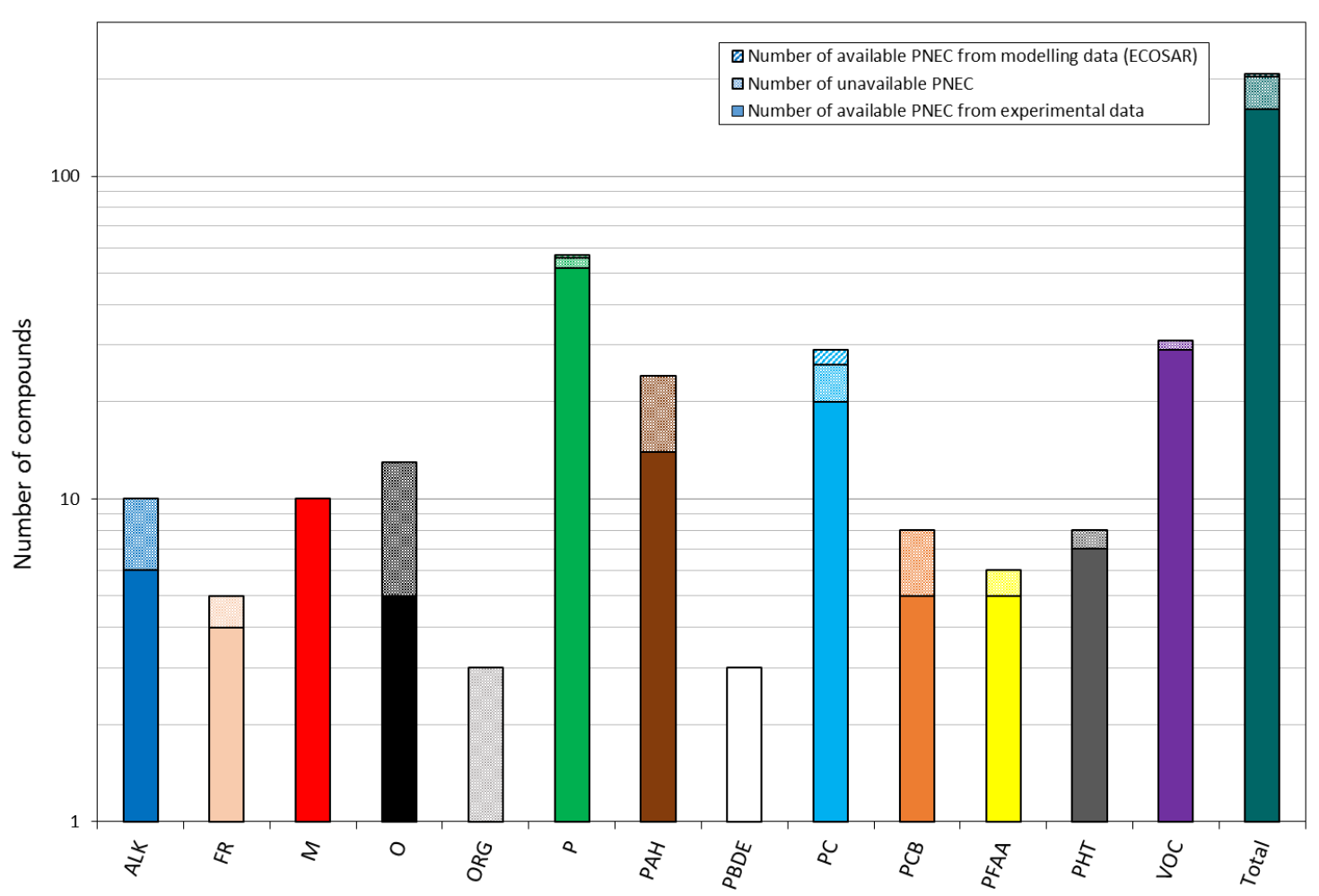

Figure 1: Distribution of the number of molecules and their PNEC available for the different families of pollutants identified in UWWDs.

The 165 PNEC values obtained have been separated into three categories according to the ranking of Orias and Perrodin (2014): a PNEC less than $0.1 \mu \mathrm{g} \mathrm{L}^{-1}$ (the most hazardous, the most ecotoxic) (Figure 2), a PNEC between 1 and $0.1 \mu \mathrm{g} \mathrm{L}^{-1}$ (intermediate ecotoxicity) (Figure 3) and a PNEC $>1 \mu \mathrm{g} \mathrm{L}^{-1}$ (less ecotoxic) (Figure 4). Finally, due to the results obtained, 59 of the compounds were ranked as very ecotoxic, 30 as ecotoxic and 76 as slightly ecotoxic.

Among the 59 very ecotoxic compounds, pesticides feature predominantly in the most hazardous category of pollutants sought in UWWDs. Indeed, 33 of the compounds with a PNEC less than $0.1 \mu \mathrm{g} \mathrm{L}^{-1}$ are pesticides. Moreover, regarding the PNEC values of a very large number of pesticides sought in UWWDs (54 compounds), they represent a potentially considerable source of hazard for this kind of matrix: 43 compounds have a PNEC value under $1.0 \mu \mathrm{g} \mathrm{L}^{-1}$ and 33 have a PNEC value under $\left.0.1 \mu \mathrm{g} \mathrm{L}^{-1}\right)$. Two other families represent a significant share of the most ecotoxic pollutants: PCs (7 compounds) and PAHs (also 7). The share of the other families is lower. The most ecotoxic compound is Endrin, a pesticide with a PNEC of $1 \cdot 22 \cdot 10^{-8} \mu \mathrm{g} \cdot \mathrm{L}^{-1}$. However, when examining slightly ecotoxic compounds (PNEC $>1 \mu \mathrm{g} \cdot \mathrm{L}^{-1}$ ), VOCs are largely represented, with 24 of the compounds listed in this

How to cite this paper: Gosset, A., Durrieu, C., Orias, F., Bayard, R., Perrodin, Y. (2017) Identification and assessment of ecotoxicological hazards attributable to pollutants in urban wet weather discharges. Environmental Science: Processes \& Impacts DOI: 10.1039/C7EM00159B 


\section{This paper is available at pubs.rsc.org}

category. The gap between the share of the other families is narrower: FR (3 compounds), PHT (6), PFAA (3), PAH (5), P (11), O (5), M (6), PC (10), ALK (1). The least toxic compound sought in UWWDs is Iohexol, a PC with a PNEC value of $45705 \mu \mathrm{g} . \mathrm{L}^{-1}$.

The large number of very ecotoxic pollutants sought in UWWDs can be explained by the fact that many substances found in UWWDs were developed to eliminate certain families of organisms, thus they have a considerable impact on many aquatic species belonging to the same family (the most relevant example is pesticides). Nevertheless, a large number of pollutants of this family present low toxicity regarding PNEC values. This observation may be explained by three possibilities: the pollutant specifically affects the organism targeted without having any effect on the others (the case of similar pesticides), it is only slightly toxic for aquatic organisms, or there are not enough data to lower the PNEC value.

We also note that no or insufficient ecotoxicity data are available for 13 of the compounds (3 PC, 6 PAH, 2 ALK, 1 ORG, 1 PFAA) detected in UWWDs. These compounds include a high number of PCs and PAHs. More ecotoxicity data are necessary for these families of compounds to avoid an underestimation of the hazard represented by UWWDs. Moreover, as it can be seen in Table 2, the PNECs of certain pollutants, such as PCBs, propylparaben and cotinine, were calculated using a small number of ecotoxicological data, and consequently their toxicity could be underestimated. However, we should bear in mind that these PNEC values were created with high safety factors (1000).

How to cite this paper: Gosset, A., Durrieu, C., Orias, F., Bayard, R., Perrodin, Y. (2017) Identification and assessment of ecotoxicological hazards attributable to pollutants in urban wet weather discharges. Environmental Science: Processes \& Impacts DOI: 10.1039/C7EM00159B 


\section{UWWD pollutant PNECs $\leq 0,1 \mu \mathrm{g} \cdot \mathrm{L}^{-1}$}

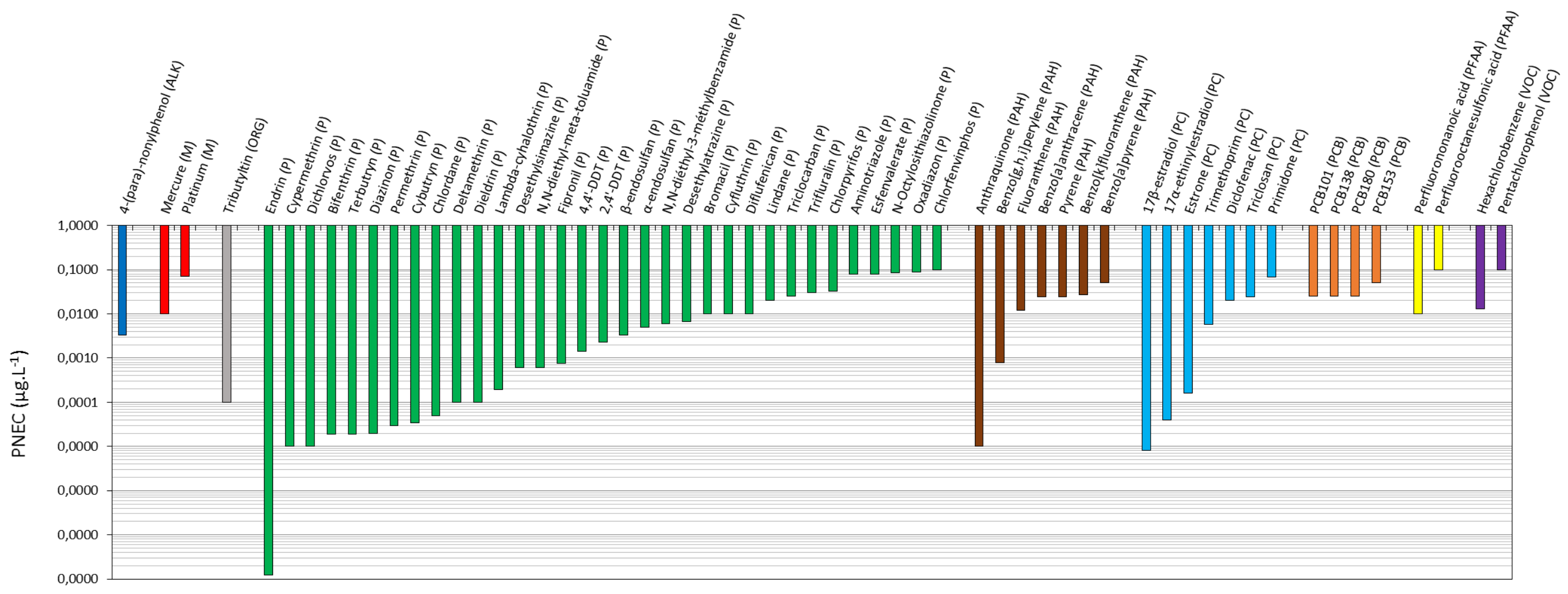

223 Figure 2: PNEC values lower or equal to $0.1 \mu \mathrm{g} \mathrm{L} \mathrm{L}^{-1}$ for the different compounds sought in UWWDs. Letters in brackets correspond to the classification in

Table 1. PNEC values are ranked in ascending order into each separated pollutant family.

How to cite this paper: Gosset, A., Durrieu, C., Orias, F., Bayard, R., Perrodin, Y. (2017) Identification and assessment of ecotoxicological hazards attributable to pollutants in urban wet weather discharges. Environmental Science: Processes \& Impacts DOI: 10.1039/C7EM00159B 


\section{$0,1 \mu \mathrm{g} \cdot \mathrm{L}^{-1}<$ UWWD pollutant PNECs $\leq 1 \mu \mathrm{g} \cdot \mathrm{L}^{-1}$}

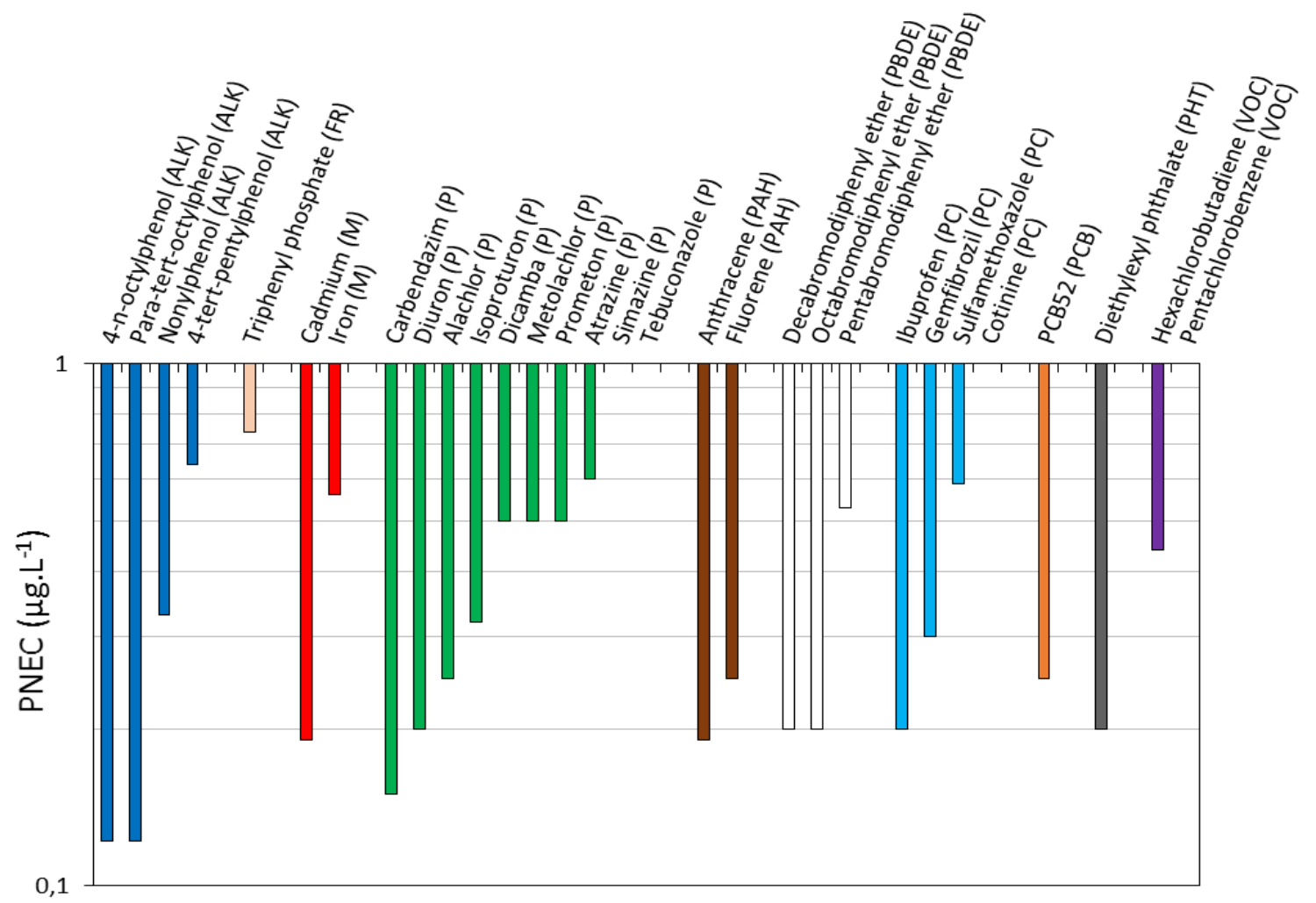

Table 1. PNEC values are ranked in ascending order into each separated pollutant family. 


\section{This paper is available at pubs.rsc.org}

\section{UWWD pollutant PNECs $>1 \mu \mathrm{g} \cdot \mathrm{L}^{-1}$}

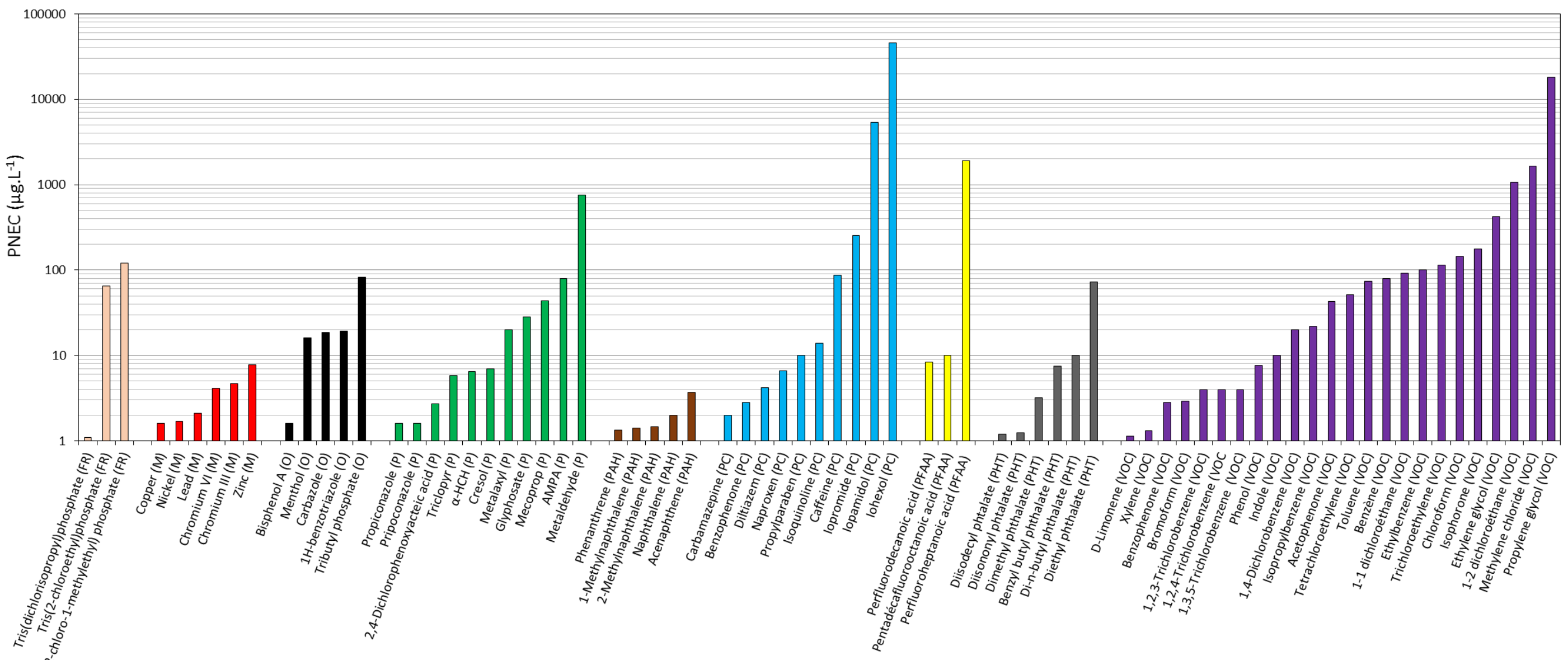

229 Figure 4: PNEC > $1 \mu \mathrm{g} \mathrm{L}{ }^{-1}$ for the different compounds sought in UWWDs. Letters in brackets correspond to the classification in Table 1. PNEC values are 


\section{This paper is available at pubs.rsc.org}

Table 2: Summary of ecotoxicity data and PNEC formulated by calculation or modelling for 60 pollutants sought in UWWDs. (Legend: D: Decomposers; P:

Primary producers; 1C: Primary consumers; 2C: Secondary consumers; C: ecotoxicity data available for organism community bioassays; Bc: Brachionus calyciflorus; EF: Extrapolation factor).

\begin{tabular}{|c|c|c|c|c|c|c|c|c|c|c|c|c|c|c|c|c|c|c|}
\hline \multirow{3}{*}{$\mathrm{N}^{\circ} \mathrm{CAS}$} & \multirow{3}{*}{ Compound } & \multirow{3}{*}{ Family } & \multicolumn{9}{|c|}{ Experimental ecotoxicity } & \multicolumn{4}{|c|}{ Data used for PNEC calculation } & \multirow{3}{*}{$\mathrm{EF}$} & \multirow{3}{*}{ PNEC (ug/L) } & \multirow{3}{*}{ Ref. } \\
\hline & & & \multirow{2}{*}{$\begin{array}{c}\text { Test } \\
\text { species }\end{array}$} & \multicolumn{4}{|c|}{ Trophic level } & \multicolumn{4}{|c|}{ Chronic test } & \multirow{2}{*}{ Specie } & \multirow{2}{*}{ Parameter } & \multirow{2}{*}{ Endpoint } & \multirow{2}{*}{ Value (ug/L) } & & & \\
\hline & & & & $\mathrm{D}$ & $P$ & $1 \mathrm{C}$ & $2 \mathrm{C}$ & $\mathrm{D}$ & $\mathrm{P}$ & 1C & $2 \mathrm{C}$ & & & & & & & \\
\hline $\begin{array}{l}104-40- \\
5\end{array}$ & 4-(para)-nonylphenol & ALK & $77+\mathrm{C}$ & 0 & 5 & 19 & $\begin{array}{c}36+17 \\
(\mathrm{Bc})\end{array}$ & 0 & 5 & 6 & $\begin{array}{c}11+17 \\
(\mathrm{Bc})\end{array}$ & Pimephales promelas & Reproduction & LOEC & $3.31 \mathrm{E}-02$ & 10 & $3.31 \mathrm{E}-03$ & 35 \\
\hline $80-46-6$ & 4-tert-pentylphenol & ALK & 4 & 0 & 0 & 1 & 3 & 0 & 0 & 1 & 2 & Danio rerio & Development & LOEC & $3.20 \mathrm{E}+01$ & 50 & $6.40 \mathrm{E}-01$ & 35 \\
\hline $78-51-3$ & $\begin{array}{c}\operatorname{Tris}(2- \\
\text { butoxyethyl)phosphate }\end{array}$ & FR & 3 & 0 & 0 & 0 & 3 & 0 & 0 & 0 & 0 & Danio rerio & Mortality & NOEC & $4.00 \mathrm{E}+03$ & & & 35 \\
\hline $\begin{array}{c}7439- \\
89-6 \\
\end{array}$ & Iron & M & 6 & 0 & 1 & 2 & 3 & 0 & 1 & 0 & 0 & Cyprinus carpio & Mortality & LC50 & $5.60 \mathrm{E}+02$ & 1000 & $5.60 \mathrm{E}-01$ & 35 \\
\hline $86-74-8$ & Carbazole & $\mathrm{O}$ & 4 & 0 & 1 & 2 & 1 & 0 & 1 & 1 & 0 & Pimephales promelas & Mortality & LC50 & $9.30 \mathrm{E}+02$ & 50 & $1.86 \mathrm{E}+01$ & 35 \\
\hline $\begin{array}{l}\text { 789-02- } \\
6\end{array}$ & 2,4'-DDT & $\mathrm{P}$ & 4 & 0 & 0 & 0 & 4 & 0 & 0 & 0 & 2 & Oryzias latipes & Reproduction (fertility) & LOEC & $2.30 \mathrm{E}-01$ & 100 & $2.30 \mathrm{E}-03$ & 35 \\
\hline $50-29-3$ & 4,4'-DDT & $\mathrm{P}$ & 40 & 0 & 11 & 21 & 8 & 0 & 11 & 5 & 0 & Hyallela azteca & Mortality & LC50 & $7.00 \mathrm{E}-02$ & 50 & $1.40 \mathrm{E}-03$ & 35 \\
\hline $\begin{array}{c}309-00- \\
2\end{array}$ & Aldrin & $\mathrm{P}$ & 55 & 0 & 0 & 18 & 37 & 0 & 0 & 0 & 0 & Paratelphusa jacquemontii & Mortality & LC50 & $9.70 \mathrm{E}-02$ & & & 35 \\
\hline $\begin{array}{l}\text { 218-01- } \\
9\end{array}$ & Chrysene & $\mathrm{P}$ & 3 & 0 & 0 & 2 & 1 & 0 & 0 & 0 & 0 & Aedes aegypti & Mortality & LC50 & $1.70 \mathrm{E}+03$ & & & 35 \\
\hline $\begin{array}{l}1319- \\
77-3\end{array}$ & Cresol & $\mathrm{P}$ & 6 & 0 & 1 & 2 & 3 & 0 & 1 & 0 & 0 & Gammarus fasciatus & Immobilization & EC50 & $7.00 \mathrm{E}+03$ & 1000 & $7.00 \mathrm{E}+00$ & 35 \\
\hline $\begin{array}{l}52315- \\
07-8\end{array}$ & Cypermethrin & $\mathrm{P}$ & 68 & 0 & 6 & 28 & $\begin{array}{l}33+1 \\
(\mathrm{Bc})\end{array}$ & 0 & 6 & 7 & $\begin{array}{l}2+1 \\
(\mathrm{Bc})\end{array}$ & Palaemonetes argentinus & Mortality & LOEC & $1.00 \mathrm{E}-04$ & 10 & $1.00 \mathrm{E}-05$ & 35 \\
\hline $\begin{array}{c}6190- \\
65-4\end{array}$ & Desethylatrazine & $\mathrm{P}$ & 11 & 0 & 8 & 3 & 0 & 0 & 7 & 1 & 0 & Stagnicola elodes & Mortality & NOEC & $3.30 \mathrm{E}-01$ & 50 & $6.60 \mathrm{E}-03$ & 35 \\
\hline $\begin{array}{l}1007- \\
28-9\end{array}$ & Desethylsimazine & $\mathrm{P}$ & 10 & 0 & 7 & 2 & 1 & 0 & 7 & 0 & 0 & Pseudokirchneriella subcapitata & Growth & NOEC & $6.00 \mathrm{E}-01$ & 1000 & $6.00 \mathrm{E}-04$ & 35 \\
\hline $\begin{array}{c}333-41- \\
5\end{array}$ & Diazinon & $\mathrm{P}$ & 131 & 0 & 9 & 53 & 69 & 0 & 9 & 8 & 18 & Daphnia magna & Reproduction & EC50 & $2.00 \mathrm{E}-04$ & 10 & $2.00 \mathrm{E}-05$ & 35 \\
\hline $60-57-1$ & Dieldrin & $\mathrm{P}$ & $98+\mathrm{C}$ & 0 & 7 & 43 & $\begin{array}{l}45+1 \\
(\mathrm{Bc})\end{array}$ & 0 & 7 & 2 & $\begin{array}{l}1+1 \\
(\mathrm{Bc})\end{array}$ & Brachionus calyciflorus & Population growth & LOEC & $1.00 \mathrm{E}-03$ & 10 & $1.00 \mathrm{E}-04$ & 35 \\
\hline $72-20-8$ & Endrin & $\mathrm{P}$ & 89 & 0 & 1 & 22 & 66 & 0 & 1 & 0 & 1 & Asellus aquaticus & Mortality & LC50 & $6.10 \mathrm{E}-07$ & 50 & $1.22 \mathrm{E}-08$ & 35 \\
\hline
\end{tabular}

How to cite this paper: Gosset, A., Durrieu, C., Orias, F., Bayard, R., Perrodin, Y. (2017) Identification and assessment of ecotoxicological hazards attributable to pollutants in urban wet weather discharges. Environmental Science: Processes \& Impacts DOI: 10.1039/C7EM00159B 
This paper is available at pubs.rsc.org

\begin{tabular}{|c|c|c|c|c|c|c|c|c|c|c|c|c|c|c|c|c|c|c|}
\hline $\begin{array}{l}2682- \\
20-4\end{array}$ & Methylisothiazolinone & $P$ & 3 & 0 & 0 & 1 & 2 & 0 & 0 & 0 & 0 & Oncorhynchus mykiss & Mortality & LC50 & $7.00 \mathrm{E}+01$ & & & 35 \\
\hline $\begin{array}{c}51218- \\
45-2\end{array}$ & Metolachlor & $P$ & 63 & 0 & 37 & 12 & 14 & 0 & 35 & 6 & 5 & Stuckenia pectinata & Photosynthesis disturbance & LOEC & $5.00 \mathrm{E}+00$ & 10 & $5.00 \mathrm{E}-01$ & 35 \\
\hline $\begin{array}{c}134-62- \\
3\end{array}$ & $\begin{array}{c}\text { N,N-diéthyl-3- } \\
\text { méthylbenzamide }\end{array}$ & $\mathrm{P}$ & 6 & 0 & 0 & 3 & 3 & 0 & 0 & 1 & 0 & Pimephales promelas & Growth & NOEC & $6.00 \mathrm{E}-01$ & 100 & $6.00 \mathrm{E}-03$ & 35 \\
\hline $\begin{array}{c}134-62- \\
3\end{array}$ & $\begin{array}{c}\mathrm{N}, \mathrm{N} \text {-diethyl-meta- } \\
\text { toluamide }\end{array}$ & $\mathrm{P}$ & 6 & 0 & 0 & 3 & 3 & 0 & 0 & 0 & 0 & Pimephales promelas & Growth (condition index) & NOEC & $6.00 \mathrm{E}-01$ & 1000 & $6.00 \mathrm{E}-04$ & 35 \\
\hline $\begin{array}{l}26530- \\
20-1\end{array}$ & N-Octylisothiazolinone & $\mathrm{P}$ & 6 & 0 & 0 & 1 & 5 & 0 & 0 & 0 & 1 & Pimephales promelas & Growth & NOEC & $8.50 \mathrm{E}+00$ & 100 & $8.50 \mathrm{E}-02$ & 35 \\
\hline $\begin{array}{c}52645- \\
53-1 \\
\end{array}$ & Permethrine & $\mathrm{P}$ & 4 & 0 & 1 & 2 & 1 & 0 & 0 & 0 & 0 & Daphnid & Mortality & LC50 & $3.00 \mathrm{E}-02$ & 1000 & $3.00 \mathrm{E}-05$ & 38 \\
\hline $\begin{array}{c}1610- \\
18-0\end{array}$ & Prometon & $P$ & 15 & 0 & 5 & 1 & 9 & 0 & 5 & 1 & 1 & Chlorella fusca var. vacuolata & $\begin{array}{l}\text { Population growth (growth } \\
\text { rate) }\end{array}$ & EC01 & $5.00 \mathrm{E}+00$ & 10 & $5.00 \mathrm{E}-01$ & 35 \\
\hline $\begin{array}{l}886-50- \\
0\end{array}$ & Terbutryn & $\mathrm{P}$ & 21 & 0 & 9 & 2 & 10 & 0 & 6 & 0 & 0 & Craticula accomoda & Growth & EC10 & $1.90 \mathrm{E}-02$ & 1000 & $1.90 \mathrm{E}-05$ & 35 \\
\hline $\begin{array}{l}\text { 101-20- } \\
2\end{array}$ & Triclocarban & $\mathrm{P}$ & 11 & 0 & 1 & 5 & 5 & 0 & 1 & 2 & 1 & Daphnia magna & Reproduction & NOEC & $2.50 \mathrm{E}-01$ & 10 & $2.50 \mathrm{E}-02$ & 35 \\
\hline $\begin{array}{l}319-84- \\
\quad 6\end{array}$ & $\alpha-\mathrm{HCH}$ & $\mathrm{P}$ & 21 & 0 & 1 & 8 & 12 & 0 & 1 & 2 & 4 & Lymnaea stagnalis & Reproduction & EC50 & $6.50 \mathrm{E}+01$ & 10 & $6.50 \mathrm{E}+00$ & 35 \\
\hline $\begin{array}{c}33213- \\
65-9\end{array}$ & $\beta$-endosulfan & $\mathrm{P}$ & 15 & 1 & 0 & 6 & 8 & 0 & 0 & 0 & 0 & Oncorhynchus mykiss & Mortality & LC50 & $3.30 \mathrm{E}+00$ & 1000 & $3.30 \mathrm{E}-03$ & 35 \\
\hline $\begin{array}{c}319-85- \\
\quad 7 \\
\end{array}$ & $\beta-\mathrm{HCH}$ & $\mathrm{P}$ & 4 & 0 & 0 & 0 & 4 & 0 & 0 & 0 & 0 & Oryzias latipes & $\begin{array}{c}\text { Behavioural changes } \\
\text { (general) }\end{array}$ & NOEC & $3.20 \mathrm{E}+01$ & & & 35 \\
\hline $90-12-0$ & 1-Methylnaphthalene & PAH & 6 & 0 & 3 & 1 & 2 & 0 & 1 & 0 & 0 & Daphnia magna & Immobilization & EC50 & $1.42 \mathrm{E}+03$ & 1000 & $1.42 \mathrm{E}+00$ & 35 \\
\hline $91-57-6$ & 2-Methylnaphthalene & PAH & 5 & 0 & 2 & 1 & 2 & 0 & 0 & 0 & 0 & Oncorhynchus mykiss & Mortality & EC50 & $1.46 \mathrm{E}+03$ & 1000 & $1.46 \mathrm{E}+00$ & 35 \\
\hline $84-65-1$ & Anthraquinone & PAH & 6 & 0 & 2 & 2 & 2 & 0 & 2 & 0 & 0 & Lemna gibba & Photosynthesis & IC50 & $1.00 \mathrm{E}-02$ & 1000 & $1.00 \mathrm{E}-05$ & 35 \\
\hline $\begin{array}{l}132-65- \\
0\end{array}$ & Dibenzothiophene & PAH & 3 & 0 & 0 & 1 & 2 & 0 & 0 & 0 & 0 & Daphnia magna & Mortality & LC50 & $4.20 \mathrm{E}+02$ & & & 35 \\
\hline $\begin{array}{l}\text { 119-61- } \\
9\end{array}$ & Benzophenone & PC & 5 & 0 & 0 & 4 & 1 & 0 & 0 & 0 & 1 & Daphnia magna & Immobilization & EC50 & $2.80 \mathrm{E}+02$ & 100 & $2.80 \mathrm{E}+00$ & 35 \\
\hline $\begin{array}{l}\text { 486-56- } \\
6\end{array}$ & Cotinine & $\mathrm{PC}$ & 1 & 0 & 1 & 0 & 0 & 0 & 1 & 0 & 0 & Lemna gibba & Biomass & NOEC & $1.00 \mathrm{E}+03$ & 1000 & $1.00 \mathrm{E}+00$ & 35 \\
\hline $\begin{array}{c}42399- \\
41-7\end{array}$ & Diltiazem & PC & 3 & 0 & 1 & 1 & 1 & 0 & 0 & 0 & 0 & Paspalum vaginatum & Calcium uptake & LOEC & $4.16 \mathrm{E}+03$ & 1000 & $4.16 \mathrm{E}+00$ & 35 \\
\hline $58-73-1$ & Diphenhydramine & PC & 2 & 0 & 0 & 1 & 1 & 0 & 0 & 1 & 0 & Ceriodaphnia dubia & Reproduction & LC50 & $3.94 \mathrm{E}+03$ & 100 & $3.94 \mathrm{E}+01$ & 35 \\
\hline $\begin{array}{l}119-65- \\
3\end{array}$ & Isoquinoline & PC & 5 & 0 & 1 & 2 & 2 & 0 & 1 & 0 & 0 & Poecilia reticulata & Mortality & LC50 & $1.40 \mathrm{E}+04$ & 1000 & $1.40 \mathrm{E}+01$ & 35 \\
\hline $94-13-3$ & Propylparaben & $\mathrm{PC}$ & 2 & 0 & 0 & 2 & 0 & 0 & 0 & 1 & 0 & Daphnia magna & Immobilization & NOEC & $1.00 \mathrm{E}+03$ & 100 & $1.00 \mathrm{E}+01$ & 35 \\
\hline
\end{tabular}

How to cite this paper: Gosset, A., Durrieu, C., Orias, F., Bayard, R., Perrodin, Y. (2017) Identification and assessment of ecotoxicological hazards attributable to pollutants in urban wet weather discharges. Environmental Science: Processes \& Impacts DOI: 10.1039/C7EM00159B 
This paper is available at pubs.rsc.org

\begin{tabular}{|c|c|c|c|c|c|c|c|c|c|c|c|c|c|c|c|c|c|c|}
\hline $\begin{array}{c}37680- \\
73-2\end{array}$ & PCB101 & РСВ & 4 & 0 & 0 & 3 & 1 & 0 & 0 & 0 & 1 & Pimephales promelas & Growth & LOEC & $2.50 \mathrm{E}+00$ & 100 & $2.50 \mathrm{E}-02$ & 35 \\
\hline $\begin{array}{l}35065- \\
28-2\end{array}$ & PCB138 & РСВ & 2 & 0 & 0 & 0 & 2 & 0 & 0 & 0 & 1 & Pimephales promelas & Growth & LOEC & $2.50 \mathrm{E}+00$ & 100 & $2.50 \mathrm{E}-02$ & 35 \\
\hline $\begin{array}{c}35065- \\
27-1 \\
\end{array}$ & PCB153 & РСB & 4 & 0 & 0 & 1 & 3 & 0 & 0 & 1 & 1 & Pimephales promelas & Growth & LOEC & $2.50 \mathrm{E}+00$ & 50 & $5.00 \mathrm{E}-02$ & 35 \\
\hline $\begin{array}{c}35065- \\
29-3\end{array}$ & PCB 180 & РСВ & 1 & 0 & 0 & 0 & 1 & 0 & 0 & 0 & 1 & Pimephales promelas & Growth & LOEC & $2.50 \mathrm{E}+00$ & 100 & $2.50 \mathrm{E}-02$ & 35 \\
\hline $\begin{array}{l}35693- \\
99-3\end{array}$ & PCB52 & РСВ & 2 & 0 & 0 & 1 & 1 & 0 & 0 & 0 & 1 & Pimephales promelas & Reproduction & NOEC & $2.50 \mathrm{E}+01$ & 100 & $2.50 \mathrm{E}-01$ & 35 \\
\hline $\begin{array}{c}335-67- \\
1\end{array}$ & $\begin{array}{c}\text { Pentadécafluorooctanoic } \\
\text { acid }\end{array}$ & PFAA & 12 & 0 & 2 & 3 & $\begin{array}{l}6+1 \\
(\mathrm{Bc})\end{array}$ & 0 & 2 & 2 & 2 & Oryzias latipes & Mortality & LOEC & $1.00 \mathrm{E}+02$ & 10 & $1.00 \mathrm{E}+01$ & 35 \\
\hline $\begin{array}{l}335-76- \\
2\end{array}$ & Perfluorodecanoic acid & PFAA & 3 & 0 & 1 & 1 & 1 & 0 & 1 & 0 & 0 & Danio rerio & Mortality & LC50 & $8.40 \mathrm{E}+03$ & 1000 & $8.40 \mathrm{E}+00$ & 35 \\
\hline $\begin{array}{l}375-85- \\
\quad 9\end{array}$ & Perfluoroheptanoic acid & PFAA & 1 & 0 & 1 & 0 & 0 & 0 & 1 & 0 & 0 & Chlorella vulgaris & Growth & EC50 & $1.90 \mathrm{E}+06$ & 1000 & $1.90 \mathrm{E}+03$ & 35 \\
\hline $\begin{array}{l}375-95- \\
\quad 1\end{array}$ & Perfluorononanoic acid & PFAA & 4 & 0 & 2 & 1 & 1 & 0 & 2 & 0 & 0 & Danio rerio & Growth & LOEC & $1.00 \mathrm{E}+01$ & 1000 & $1.00 \mathrm{E}-02$ & 35 \\
\hline $\begin{array}{l}1763- \\
23-1 \\
\end{array}$ & $\begin{array}{l}\text { Perfluorooctanesulfonic } \\
\text { acid }\end{array}$ & PFAA & 4 & 0 & 0 & 2 & 2 & 0 & 0 & 1 & 0 & Oryzias latipes & Mortality & LOEC & $1.00 \mathrm{E}+01$ & 100 & $1.00 \mathrm{E}-01$ & 35 \\
\hline $\begin{array}{c}2058- \\
94-8 \\
\end{array}$ & $\begin{array}{c}\text { Perfluoroundecanoic } \\
\text { acid }\end{array}$ & PFAA & 2 & 0 & 0 & 1 & 1 & 0 & 0 & 0 & 0 & Daphnia magna & Immobilization & NOEC & $5.64 \mathrm{E}+04$ & & & 35 \\
\hline $\begin{array}{l}117-81- \\
7\end{array}$ & Diethylexyl phthalate & PHT & 29 & 0 & 3 & 5 & 21 & 0 & 3 & 2 & 3 & Danio rerio & Reproduction & NOEC & $2.00 \mathrm{E}+00$ & 10 & $2.00 \mathrm{E}-01$ & 35 \\
\hline $\begin{array}{c}26761- \\
40-0\end{array}$ & Diisodecyl phtalate & PHT & 7 & 0 & 1 & 1 & 5 & 0 & 1 & 1 & 0 & Daphnia magna & Mortality & LOEC & $6.00 \mathrm{E}+01$ & 50 & $1.20 \mathrm{E}+00$ & 35 \\
\hline $\begin{array}{c}27554- \\
26-3\end{array}$ & Diisononyl phtalate & PHT & 7 & 0 & 1 & 2 & 4 & 0 & 1 & 1 & 0 & Daphnia magna & Reproduction & NOEC & $6.20 \mathrm{E}+01$ & 50 & $1.24 \mathrm{E}+00$ & 35 \\
\hline $\begin{array}{c}131-11- \\
3 \\
\end{array}$ & Dimethyl phthalate & PHT & 9 & 0 & 1 & 2 & 6 & 0 & 1 & 0 & 0 & Cyprinodon variegatus & Mortality & NOEC & $3.20 \mathrm{E}+03$ & 1000 & $3.20 \mathrm{E}+00$ & 35 \\
\hline 98-86-2 & Acetophenone & VOC & 4 & 0 & 1 & 2 & 1 & 0 & 0 & 0 & 0 & Tetrahymena pyriformis & Population Growth & EC50 & $4.28 \mathrm{E}+04$ & 1000 & $4.28 \mathrm{E}+01$ & 35 \\
\hline $\begin{array}{l}\text { 119-61- } \\
9\end{array}$ & Benzophenone & VOC & 5 & 0 & 0 & 4 & 1 & 0 & 0 & 1 & 0 & Daphnia magna & Immobilization & EC50 & $2.80 \mathrm{E}+02$ & 100 & $2.80 \mathrm{E}+00$ & 35 \\
\hline $75-25-2$ & Bromoform & VOC & 5 & 0 & 1 & 2 & 2 & 0 & 1 & 0 & 0 & Cyprinodon variegatus & Mortality & NOEC & $2.90 \mathrm{E}+03$ & 1000 & $2.90 \mathrm{E}+00$ & 35 \\
\hline $\begin{array}{l}138-86- \\
3\end{array}$ & D-Limonene & VOC & 11 & 0 & 1 & 7 & 3 & 0 & 1 & 0 & 0 & Lymnaea acuminata & Mortality & LC50 & $1.13 \mathrm{E}+03$ & 1000 & $1.13 \mathrm{E}+00$ & 35 \\
\hline $\begin{array}{c}107-21- \\
1\end{array}$ & Ethylene glycol & VOC & 20 & 1 & 1 & 7 & 11 & 0 & 1 & 1 & 1 & Ceriodaphnia dubia & Reproduction & NOEC & $4.20 \mathrm{E}+03$ & 10 & $4.20 \mathrm{E}+02$ & 35 \\
\hline $\begin{array}{l}120-72- \\
9\end{array}$ & Indole & VOC & 3 & 0 & 1 & 2 & 0 & 0 & 1 & 1 & 0 & Oryzias latipes & Mortality & LC50 & $1.00 \mathrm{E}+03$ & 100 & $1.00 \mathrm{E}+01$ & 35 \\
\hline $\begin{array}{l}124-76- \\
5\end{array}$ & Isoborneol & VOC & 1 & 0 & 0 & 0 & 1 & 0 & 0 & 0 & 0 & Fish & Mortality & LC50 & $5.90 \mathrm{E}+04$ & & & 38 \\
\hline
\end{tabular}

How to cite this paper: Gosset, A., Durrieu, C., Orias, F., Bayard, R., Perrodin, Y. (2017) Identification and assessment of ecotoxicological hazards attributable to pollutants in urban wet weather discharges. Environmental Science: Processes \& Impacts DOI: 10.1039/C7EM00159B 


\section{This paper is available at pubs.rsc.org}

\section{$\underline{3.3 \text { Concentration of pollutants in separate and combined systems }}$}

Maximum pollutant concentrations found in UWWDs were assessed using available literature and are presented in Tables 3 and 4. MECmax were very variable, with some pollutants (e.g. hexachlorobenzene) found at several ng.L $\mathrm{L}^{-1}$, while others (e.g. copper) were found at a few mg. $\mathrm{L}^{-1}$. However, the compound concentrations generally ranged between ng. $\mathrm{L}^{-1}$ and several hundred $\mu \mathrm{g} . \mathrm{L}^{-1}$.

On one hand, as observed in Table 3, 61 compounds were detected in stormwater samples, and 50 were sought but not detected. We found the highest concentrations for zinc $\left(2.12 \mathrm{mg} . \mathrm{L}^{-1}\right)$. High concentrations of pollutants such as other heavy metals (copper, nickel) and pesticides (such as glyphosate and AMPA) and PAH (e.g. pyrene) were also detected. The main families of pollutants detected at high concentrations were PAH, VOC, ALK, PHT, P and M.

On the other hand, out of the 81 chemicals of interest sought in the dissolved part of the CSO samples, 49 were reported (see Table 4). The highest concentration was obtained for zinc $\left(236 \mu \mathrm{g} . \mathrm{L}^{-1}\right)$. The main families found at high concentrations were M, P and PC. The occurrence of high concentrations of heavy metals in separate systems and CSO is not surprising due to the source of wastewater and rainfall runoff. More precisely, tyre wear, vehicle brake deposition, conveyance system infrastructure (e.g. downspouts and culverts), and atmospheric deposition can lead to the presence of heavy metals. ${ }^{4}$ Moreover, the remobilization of wastewater and in-sewer deposits (due to pollutant water/particle partition) can represent a large share of the pollutant load in CSO, such as $\mathrm{Cu}$ and PAH. ${ }^{58}$ Launay et al. ${ }^{64}$ observed the same phenomenon for pharmaceutical compounds and industrial chemicals. Contrary to stormwater, we note the presence of PC compounds sought and detected in the dissolved phase (18 compounds),

If we compare the Maximum concentrations of UWWD pollutants with other urban effluents (e.g. urban wastewater and hospital wastewater), such as phthalates, heavy metals, pharmaceutical compounds and pesticides, $\mathrm{MEC}_{\max }$ were close to those detected in these other matrices. ${ }^{60,61,62,63}$ Some of them, such as Bisphenol A, concentrations in UWWDs (max. $130 \mu \mathrm{g} \mathrm{L}^{-1}$ ) can be even higher than in wastewater (max. $2.14 \mu \mathrm{g} \mathrm{L}^{-1}$ ). This fact was also observed with heavy metals such as copper and zinc. Consequently, since they undergo hardly any or no treatment, and that most of few current treatment How to cite this paper: Gosset, A., Durrieu, C., Orias, F., Bayard, R., Perrodin, Y. (2017) Identification and assessment of ecotoxicological hazards attributable to pollutants in urban wet weather discharges. Environmental Science: Processes \& Impacts DOI: 10.1039/C7EM00159B 


\section{This paper is available at pubs.rsc.org}

263

methods employed do not reduce concentration of many identified pollutants, considerable amounts of these substances may enter surface waters.

Nevertheless, it is important to point out that the data collected and discussed above have some limitations. Firstly, few studies have analyzed the dissolved phase of discharges and thus many compounds identified and sought in UWWDs are not taken into account here because they are found in only the particle or total phase (due to their low solubility and high affinity for the particle phase). Consequently, the number of compounds detected here is limited (see Table 3 and Table 4). Secondly, concentrations found in UWWDs need to be considered with caution because, as stated in the study of Huber et al., ${ }^{52}$ concentrations measured in stormwater samples can be influenced by different parameters such as sample collection, preparation and analysis, which can also affect the concentrations observed in discharges. 
This paper is available at pubs.rsc.org

Table 3: Maximum Measured Effluent Concentrations (MECmax) of micropollutants in the dissolved part sought in stormwater samples (ND: Not detected, *:

287 Unspecified).

\begin{tabular}{|c|c|c|c|c|c|c|c|c|c|}
\hline $\begin{array}{l}\text { Pollutant } \\
\text { family }\end{array}$ & Pollutant & $\begin{array}{l}\mathrm{MECmax} \\
(\mu \mathrm{g} / \mathrm{L})\end{array}$ & Country & Ref. & $\begin{array}{c}\text { Pollutant } \\
\text { family }\end{array}$ & Pollutant & $\begin{array}{c}\mathrm{MECmax} \\
(\mu \mathrm{g} / \mathrm{L})\end{array}$ & Country & Ref. \\
\hline ALK & $\begin{array}{l}\text { 4-n-octylphenol } \\
\text { (ALK) }\end{array}$ & ND & France & 4 & PAH & Dibenzothiophene (PAH) & 0.0417 & USA (Washington D.C.) & 2 \\
\hline ALK & $\begin{array}{l}\text { 4-nonylphenol di- } \\
\text { ethoxylate (ALK) }\end{array}$ & ND & France & 4 & PAH & Acenaphthylene (PAH) & 0.070 & $\begin{array}{c}\text { Australia; France; Sweden; USA } \\
\text { (Alabama); USA (Washington D.C.) }\end{array}$ & $\begin{array}{l}2 ; 4 ; 5 \\
; 47 ; 48\end{array}$ \\
\hline ALK & $\begin{array}{l}\text { 4-tert-pentylphenol } \\
\text { (ALK) }\end{array}$ & 0.7 & Sweden & 5 & PAH & Perylène (PAH) & 0.12 & USA (Washington D.C.) & 2 \\
\hline ALK & $\begin{array}{c}\text { Para-tert- } \\
\text { octylphenol (ALK) }\end{array}$ & 0.99 & France, Sweden & $4 ; 5$ & PAH & $\begin{array}{c}\text { Dibenzo[a,h]anthracene } \\
\text { (PAH) }\end{array}$ & 0.13 & $\begin{array}{c}\text { France; Sweden; USA (Alabama); } \\
\text { USA (Washington D.C.) }\end{array}$ & $\begin{array}{l}2 ; 4 ; \\
5 ; 47\end{array}$ \\
\hline ALK & $\begin{array}{l}\text { 4-Octylphenol } \\
\text { monoethoxylate } \\
\text { (ALK) }\end{array}$ & 4.39 & Sweden & 5 & PAH & Benzo[a]anthracene $(\mathrm{PAH})$ & 0.34 & $\begin{array}{c}\text { Australia; France; Sweden; USA } \\
\text { (Alabama); USA (South Carolina); } \\
\text { USA (Washington D.C.) }\end{array}$ & $\begin{array}{l}2 ; 4 ; \\
5 ; 47 \\
48 ; 49\end{array}$ \\
\hline ALK & $\begin{array}{l}\text { 4-(para)- } \\
\text { nonylphenol } \\
\text { (ALK) }\end{array}$ & 7.7 & France, Sweden & $4 ; 5$ & PAH & $\begin{array}{l}\text { Benzo[k]fluoranthene } \\
\text { (PAH) }\end{array}$ & 0.40 & $\begin{array}{c}\text { France; Sweden; USA (Alabama); } \\
\text { USA (Washington D.C.) }\end{array}$ & $\begin{array}{l}2 ; 4 ; \\
5 ; 47\end{array}$ \\
\hline ALK & $\begin{array}{l}\text { 4-tert-butylphenol } \\
\text { (ALK) }\end{array}$ & 7.82 & France, Sweden & $4 ; 5$ & PAH & Benzo[e]pyrene (PAH) & 0.42 & $\begin{array}{l}\text { USA (South Carolina); USA } \\
\text { (Washington D.C.) }\end{array}$ & $2 ; 48$ \\
\hline ALK & $\begin{array}{l}\text { 4-nonylphenol } \\
\text { mono-ethoxylate } \\
\text { (ALK) }\end{array}$ & 8.36 & Sweden & 5 & PAH & $\begin{array}{c}\text { Indeno[1,2,3-cd }] \text { pyrene } \\
\text { (PAH) }\end{array}$ & 505 & $\begin{array}{c}\text { Australia; France; Sweden; USA } \\
\text { (Alabama); USA (South Carolina); } \\
\text { USA (Washington D.C.) }\end{array}$ & $\begin{array}{l}2 ; 4 ; \\
5 ; 47 \\
48 ; 49\end{array}$ \\
\hline M & Platinium (M) & ND & France & 4 & PAH & Benzo[a]pyrene (PAH) & 0.54 & $\begin{array}{c}\text { Australia; France; Sweden; USA } \\
\text { (Alabama); USA (South Carolina); } \\
\text { USA (Washington D.C.) }\end{array}$ & $\begin{array}{l}2 ; 4 ; \\
5 ; 47 \\
48 ; 49\end{array}$ \\
\hline M & Mercury (M) & 0.01 & Canada; France & $4 ; 41$ & PAH & Benzo[g,h,i]perylene (PAH) & 0.55 & $\begin{array}{c}\text { Australia; Sweden; USA (Alabama); } \\
\text { USA (South Carolina); USA } \\
\text { (Washington D.C.) }\end{array}$ & $\begin{array}{l}2 ; 5 ; \\
47 ; 48 \\
\quad 49\end{array}$ \\
\hline M & Lead (M) & 15.9 & $\begin{array}{l}\text { Australia; China; France; } \\
\text { USA (Alabama); USA } \\
\text { (California); * }\end{array}$ & $\begin{array}{c}4 ; 5 ; 47 ; \\
50 ; 51 ; 52\end{array}$ & PAH & Anthracene (PAH) & 0.56 & $\begin{array}{c}\text { Australia; France; Sweden; USA } \\
\text { (Alabama); USA (South Carolina); } \\
\text { USA (Washington D.C.) }\end{array}$ & $\begin{array}{l}2 ; 4 ; \\
5 ; 47 \\
48 ; 49\end{array}$ \\
\hline M & Cadmium (M) & 31.1 & $\begin{array}{l}\text { Australia; France; USA } \\
\text { (Alabama); USA } \\
\text { (California); }{ }^{*}\end{array}$ & $\begin{array}{l}4 ; 47 ; 50 ; \\
52 ; 55\end{array}$ & PAH & Chrysene (PAH) & 0.62 & $\begin{array}{c}\text { Australia; France; Sweden; USA } \\
\text { (Alabama); USA (South Carolina); } \\
\text { USA (Washington D.C.) }\end{array}$ & $\begin{array}{l}2 ; 4 ; \\
5 ; 47 \\
48 ; 49\end{array}$ \\
\hline M & Chromium (M) & 32 & $\begin{array}{l}\text { Australia; France; USA } \\
\text { (Alabama); USA } \\
\text { (California);* }\end{array}$ & $\begin{array}{l}4 ; 47 ; 50 \\
\quad 52 ; 55\end{array}$ & PAH & Acenaphthene (PAH) & 0.72 & $\begin{array}{l}\text { Australia; France; Sweden; USA } \\
\text { (Alabama) USA (Washington D.C.) }\end{array}$ & $\begin{array}{c}4 ; 5 ; \\
47 ; 49\end{array}$ \\
\hline M & Nickel (M) & 49 & $\begin{array}{l}\text { France; USA (Alabama); } \\
\text { USA (California); } *\end{array}$ & $\begin{array}{c}4 ; 5 ; 47 ; \\
52\end{array}$ & PAH & $\begin{array}{l}\text { Benzo[b]fluoranthene } \\
\text { (PAH) }\end{array}$ & 0.98 & $\begin{array}{l}\text { Australia; France; USA (Alabama); } \\
\text { USA (South Carolina); USA } \\
\text { (Washington D.C.) }\end{array}$ & $\begin{array}{c}2 ; 4 ; \\
47 ; 48 \\
\quad 49\end{array}$ \\
\hline
\end{tabular}

How to cite this paper: Gosset, A., Durrieu, C., Orias, F., Bayard, R., Perrodin, Y. (2017) Identification and assessment of ecotoxicological hazards attributable to pollutants in urban wet weather discharges. Environmental Science: Processes \& Impacts DOI: 10.1039/C7EM00159B 
This paper is available at pubs.rsc.org

\begin{tabular}{|c|c|c|c|c|c|c|c|c|c|}
\hline M & Copper (M) & 1520 & $\begin{array}{l}\text { Australia; China; France; } \\
\text { USA (Alabama); USA } \\
\text { (California); * }\end{array}$ & $\begin{array}{c}4 ; 5 ; 47 ; \\
50 ; 51 ; 52\end{array}$ & PAH & Phenanthrene (PAH) & 1.32 & $\begin{array}{c}\text { Australia; France; Sweden; USA } \\
\text { (Alabama); USA (South Carolina); } \\
\text { USA (Washington D.C.) }\end{array}$ & $\begin{array}{l}2 ; 4 ; \\
5 ; 47 \\
48 ; 49\end{array}$ \\
\hline M & Zinc (M) & 2118 & $\begin{array}{l}\text { Australia; China; France; } \\
\text { USA (Alabama); USA } \\
\text { (California); } *\end{array}$ & $\begin{array}{l}4 ; 5 ; 47 ; \\
50 ; 51 ; 52\end{array}$ & PAH & Fluorene (PAH) & 1.37 & $\begin{array}{c}\text { Australia; France; Sweden; USA } \\
\text { (Alabama); USA (South Carolina); } \\
\text { USA (Washington D.C.) }\end{array}$ & $\begin{array}{l}2 ; 4 ; \\
5 ; 47 \\
48 ; 49\end{array}$ \\
\hline $\mathrm{O}$ & Bisphenol A (O) & 130 & Sweden & 5 & PAH & Fluoranthene (PAH) & 14 & $\begin{array}{c}\text { Australia; France; Sweden; USA } \\
\text { (Alabama); USA (South Carolina); } \\
\text { USA (Washington D.C.) }\end{array}$ & $\begin{array}{l}2 ; 4 \\
5 ; 47 \\
48 ; 49\end{array}$ \\
\hline ORG & Dibutyltin (ORG) & ND & France & 4 & PAH & Naphthalene (PAH) & 17 & $\begin{array}{c}\text { Australia; France; Sweden; USA } \\
\text { (Alabama); USA (Washington D.C.) }\end{array}$ & $\begin{array}{l}2 ; 4 ; 5 \\
; 47 ; 49\end{array}$ \\
\hline ORG & Tributyltin (ORG) & ND & France & 4 & PAH & Pyrene (PAH) & 20 & $\begin{array}{c}\text { Australia; France; Sweden; USA } \\
\text { (Alabama); USA (South Carolina); } \\
\text { USA (Washington D.C.) }\end{array}$ & $\begin{array}{c}2 ; 4 ; \\
5 ; 47 \\
48 ; 49\end{array}$ \\
\hline ORG & $\begin{array}{l}\text { Monobutyltin } \\
\quad(\mathrm{ORG})\end{array}$ & 0.025 & France & 4 & PBDE & $\begin{array}{l}\text { Decabromodiphenyl ether } \\
\text { (PBDE) }\end{array}$ & ND & France & 4 \\
\hline $\mathrm{P}$ & 2,4'-DDT (P) & ND & France & 4 & PBDE & $\begin{array}{l}\text { Octabromodiphenyl ether } \\
\text { (PBDE) }\end{array}$ & ND & France & 4 \\
\hline $\mathrm{P}$ & 4,4'-DDT (P) & ND & France & 4 & PBDE & $\begin{array}{l}\text { Pentabromodiphenyl ether } \\
\text { (PBDE) }\end{array}$ & ND & France & 4 \\
\hline $\mathrm{P}$ & Alachlor (P) & ND & France & 4 & PCB & PCB101 (PCB) & ND & France & 4 \\
\hline $\mathrm{P}$ & Aldrin (P) & ND & France; USA (Alabama) & $4 ; 47$ & PCB & PCB118 (PCB) & ND & France & 4 \\
\hline $\mathrm{P}$ & Atrazine $(\mathrm{P})$ & ND & France & 4 & PCB & PCB138 (PCB) & ND & France & 4 \\
\hline $\mathrm{P}$ & Chlordane $(\mathrm{P})$ & ND & France; USA (Alabama) & $4 ; 47$ & PCB & PCB153 (PCB) & ND & France & 4 \\
\hline $\mathrm{P}$ & $\begin{array}{l}\text { Chlorfenvinphos } \\
\text { (P) }\end{array}$ & ND & France & 4 & PCB & PCB180 (PCB) & ND & France & 4 \\
\hline $\mathrm{P}$ & Chlorpyrifos (P) & ND & France & 4 & PCB & PCB194 (PCB) & ND & France & 4 \\
\hline $\mathrm{P}$ & $\begin{array}{l}\text { Desethylsimazine } \\
\text { (P) }\end{array}$ & ND & France & 4 & PCB & РCB28 (PCB) & ND & France & 4 \\
\hline $\mathrm{P}$ & Dieldrin $(\mathrm{P})$ & ND & France & 4 & PCB & PCB52 (PCB) & ND & France & 4 \\
\hline $\mathrm{P}$ & Endrin $(\mathrm{P})$ & ND & France; USA (Alabama) & $4 ; 47$ & PFAA & $\begin{array}{l}\text { Perfluoroheptanoic acid } \\
\text { (PFAA) }\end{array}$ & 0.011 & $\begin{array}{c}\text { Japan; Singapour; Switzerland; USA } \\
\text { (Minnesota) }\end{array}$ & $\begin{array}{l}42 ; 43 ; \\
44 ; 45\end{array}$ \\
\hline $\mathrm{P}$ & Esfenvalerate $(\mathrm{P})$ & ND & France; USA (Alabama) & $4 ; 47$ & PFAA & $\begin{array}{l}\text { Perfluoroundecanoic acid } \\
\text { (PFAA) }\end{array}$ & 0.045 & $\begin{array}{c}\text { Japan; Singapour; Switzerland; USA } \\
\text { (Minnesota) }\end{array}$ & $\begin{array}{c}42 ; 43 \\
44 ; 45 ; \\
46\end{array}$ \\
\hline $\mathrm{P}$ & Lindane $(\mathrm{P})$ & ND & France & 4 & PFAA & $\begin{array}{l}\text { Perfluorodecanoic acid } \\
\text { (PFAA) }\end{array}$ & 0.077 & $\begin{array}{l}\text { Japan; Singapour; Switzerland; USA } \\
\text { (Minnesota) }\end{array}$ & $\begin{array}{c}42 ; 43 ; \\
44 ; 45 ; \\
46\end{array}$ \\
\hline $\mathrm{P}$ & Simazine $(\mathrm{P})$ & ND & France & 4 & PFAA & $\begin{array}{l}\text { Perfluorononanoic acid } \\
\text { (PFAA) }\end{array}$ & 0.093 & $\begin{array}{l}\text { Japan; Singapour; Switzerland; USA } \\
\text { (Minnesota) }\end{array}$ & $\begin{array}{c}42 ; 43 \\
44 ; 45 ; \\
46\end{array}$ \\
\hline
\end{tabular}

How to cite this paper: Gosset, A., Durrieu, C., Orias, F., Bayard, R., Perrodin, Y. (2017) Identification and assessment of ecotoxicological hazards attributable to pollutants in urban wet weather discharges. Environmental Science: Processes \& Impacts DOI: 10.1039/C7EM00159B 
This paper is available at pubs.rsc.org

\begin{tabular}{|c|c|c|c|c|c|c|c|c|c|}
\hline$P$ & Trifluralin $(\mathrm{P})$ & ND & France & 4 & PFAA & $\begin{array}{l}\text { Perfluorooctanesulfonic } \\
\text { acid (PFAA) }\end{array}$ & 0.16 & $\begin{array}{c}\text { Japan; Singapour; Switzerland; USA } \\
\text { (Minnesota) }\end{array}$ & $\begin{array}{c}42 ; 43 ; \\
44 ; 45 ; \\
46\end{array}$ \\
\hline $\mathrm{P}$ & $\alpha$-endosulfan (P) & ND & France & 4 & PFAA & $\begin{array}{l}\text { Pentadecafluorooctanoic } \\
\text { acid (PFAA) }\end{array}$ & 0.17 & $\begin{array}{c}\text { Japan; Singapour; Switzerland; USA } \\
\text { (Minnesota) }\end{array}$ & $\begin{array}{c}42 ; 43 ; \\
44 ; 45 ; \\
\quad 46\end{array}$ \\
\hline $\mathrm{P}$ & $\beta$-endosulfan $(\mathrm{P})$ & ND & France & 4 & PHT & Dimethyl phthalate (PHT) & ND & USA (Alabama) & 47 \\
\hline$P$ & $\begin{array}{l}\text { Lambda- } \\
\text { cyhalothrin }(\mathrm{P})\end{array}$ & 0.007 & USA (California) & 40 & PHT & Di-n-octyl phtalate (PHT) & ND & France & 4 \\
\hline $\mathrm{P}$ & Cyfluthrin (P) & 0.023 & USA (California) & 40 & PHT & Di-n-butyl phthalate (PHT) & 1 & Sweden; USA (Alabama) & $5 ; 47$ \\
\hline $\mathrm{P}$ & Cypermethrin (P) & 0.026 & USA (California) & 40 & PHT & Diisodecyl phtalate (PHT) & 1.2 & Sweden & 5 \\
\hline$P$ & Bifenthrin $(\mathrm{P})$ & 0.034 & USA (California) & 40 & PHT & Diisononyl phtalate (PHT) & 2 & Sweden & 5 \\
\hline$P$ & Metaldehyde (P) & 0.05 & France & 4 & PHT & Diethyl phthalate (PHT) & 15.9 & Sweden; USA (Alabama) & $5 ; 47$ \\
\hline$P$ & Permethrin $(\mathrm{P})$ & 0.13 & USA (California) & 40 & PHT & $\begin{array}{l}\text { Benzyl butyl phthalate } \\
\text { (PHT) }\end{array}$ & 16 & Sweden; USA (Alabama) & $5 ; 47$ \\
\hline$P$ & Deltamethrin (P) & 0.25 & USA (California) & 40 & PHT & $\begin{array}{l}\text { Diethylhexyl phthalate } \\
\text { (PHT) }\end{array}$ & 45 & France; Sweden; USA (Alabama) & $\begin{array}{c}4 ; 5 ; \\
47\end{array}$ \\
\hline$P$ & Aminotriazole $(\mathrm{P})$ & 0.5 & France & $4 ; 47 ; 48$ & VOC & $\begin{array}{l}\text { 1,2,3-Trichlorobenzene } \\
\text { (VOC) }\end{array}$ & ND & France & 4 \\
\hline$P$ & Isoproturon (P) & 0.6 & France & 4 & VOC & $\begin{array}{l}\text { 1,2,4-Trichlorobenzene } \\
\text { (VOC) }\end{array}$ & ND & France & 4 \\
\hline$P$ & Diuron $(\mathrm{P})$ & 0.64 & France & 4 & VOC & $\begin{array}{c}1,3,5- \\
\text { Trichlorobenzene (VOC) }\end{array}$ & ND & France & 4 \\
\hline $\mathrm{P}$ & AMPA (P) & 19 & France; Switzerland & $4 ; 53 ; 54$ & VOC & 1-2 dichloroéthane (VOC) & ND & France & 4 \\
\hline$P$ & Glyphosate $(\mathrm{P})$ & 150 & France; Switzerland & $4 ; 53 ; 54$ & VOC & Benzène (VOC) & ND & France & 4 \\
\hline & & & & & VOC & Chloroform (VOC) & ND & France & 4 \\
\hline & & & & & VOC & Ethylbenzene (VOC) & ND & France & 4 \\
\hline & & & & & VOC & Hexachlorobenzene (VOC) & ND & France & 4 \\
\hline & & & & & VOC & $\begin{array}{l}\text { Hexachlorobutadiene } \\
\text { (VOC) }\end{array}$ & ND & France & 4 \\
\hline & & & & & VOC & Isopropylbenzene (VOC) & ND & France & 4 \\
\hline & & & & & VOC & Pentachlorobenzene (VOC) & ND & France & 4 \\
\hline & & & & & VOC & Tetrachloroethylene (VOC) & ND & France & 4 \\
\hline & & & & & VOC & Toluene (VOC) & ND & France & 4 \\
\hline & & & & & VOC & Trichloroethylene (VOC) & ND & France & 4 \\
\hline & & & & & VOC & Xylene (VOC) & ND & France & 4 \\
\hline & & & & & VOC & Pentachlorophenol (VOC) & 0.2 & France & 4 \\
\hline & & & & & $\mathrm{VOC}$ & Dichloromethane (VOC) & 13 & France & 4 \\
\hline & & & & & VOC & $\begin{array}{c}\text { (VOC) } \\
\end{array}$ & 47 & USA (Alabama) & 48 \\
\hline
\end{tabular}


This paper is available at pubs.rsc.org

Table 4: Maximum Measured Effluent Concentrations (MECmax) of the micropollutants sought in the dissolved part of CSO samples (ND: Not detected, *:

291 Unspecified).

\begin{tabular}{|c|c|c|c|c|c|c|c|c|c|}
\hline $\begin{array}{l}\text { Pollutant } \\
\text { family }\end{array}$ & Pollutant & $\begin{array}{l}\operatorname{MECmax} \\
(\mu \mathrm{g} / \mathrm{L})\end{array}$ & Country & Ref. & $\begin{array}{l}\text { Pollutant } \\
\text { family }\end{array}$ & Pollutant & $\begin{array}{l}\operatorname{MECmax} \\
(\mu \mathrm{g} / \mathrm{L})\end{array}$ & Country & Ref. \\
\hline ALK & 4-n-octylphenol (ALK) & ND & France & 57 & $\mathrm{PC}$ & Estrone (PC) & ND & South Korea & 12 \\
\hline ALK & Para-tert-octylphenol (ALK) & ND & France & 57 & PC & Diltiazem (PC) & ND & South Korea & 12 \\
\hline ALK & 4-tert-butylphenol (ALK) & 0.080 & France & 57 & $\mathrm{PC}$ & Trimethoprim (PC) & ND & South Korea & 12 \\
\hline ALK & Nonylphenol (ALK) & 0.390 & France & 57 & $\mathrm{PC}$ & Meprobamate (PC) & ND & South Korea & 12 \\
\hline FR & $\begin{array}{l}\text { Tris(2-chloro-1-methylethyl) } \\
\text { phosphate (FR) }\end{array}$ & 1.140 & South Korea & 12 & $\mathrm{PC}$ & Diclofenac (PC) & ND & South Korea & 12 \\
\hline M & Nickel (M) & 1.1 & France & $15 ; 57 ; 58$ & $\mathrm{PC}$ & Gemfibrozil (PC) & 0.006 & South Korea & 12 \\
\hline M & Chromium (M) & 1.8 & France & $15 ; 57 ; 58$ & $\mathrm{PC}$ & Diphenhydramine (PC) & 0.023 & South Korea & 12 \\
\hline M & Cadmium (M) & 9.9 & France, United Kingdom & $\begin{array}{c}15 ; 57 ; 58 ; \\
59\end{array}$ & $\mathrm{PC}$ & Primidone (PC) & 0.040 & South Korea & 12 \\
\hline M & Copper (M) & 65.4 & France, United Kingdom & $\begin{array}{c}15 ; 57 ; 58 ; \\
59\end{array}$ & $\mathrm{PC}$ & Triclosan (PC) & 0.055 & South Korea & 12 \\
\hline M & Lead (M) & 143.4 & France, United Kingdom & $\begin{array}{c}15 ; 57 ; 58 ; \\
59\end{array}$ & $\mathrm{PC}$ & Carbamazepine (PC) & 0.060 & South Korea & 12 \\
\hline $\mathrm{M}$ & Zinc (M) & 236.1 & France, United Kingdom & $\begin{array}{c}15 ; 57 ; 58 ; \\
59\end{array}$ & $\mathrm{PC}$ & Propylparaben (PC) & 0.069 & South Korea & 12 \\
\hline $\mathrm{O}$ & 1H-benzotriazole $(\mathrm{O})$ & 0.078 & South Korea & 12 & $\mathrm{PC}$ & Sulfamethoxazole (PC) & 0.070 & South Korea & 12 \\
\hline ORG & Dibutyltin (ORG) & $\mathrm{ND}$ & France & 57 & $\mathrm{PC}$ & Trimethoprim (PC) & 0.073 & South Korea & 12 \\
\hline ORG & Tributyltin (ORG) & $\mathrm{ND}$ & France & 57 & $\mathrm{PC}$ & Benzophenone (PC) & 0.136 & South Korea & 12 \\
\hline ORG & Monobutyltin (ORG) & 0.013 & France & 57 & $\mathrm{PC}$ & Atenolol (PC) & 0.363 & South Korea & 12 \\
\hline $\mathrm{P}$ & Aldrin $(\mathrm{P})$ & $\mathrm{ND}$ & France & 57 & $\mathrm{PC}$ & Acesulfame (PC) & 0.727 & South Korea & 12 \\
\hline$P$ & Dieldrin $(\mathrm{P})$ & $\mathrm{ND}$ & France & 57 & $\mathrm{PC}$ & Naproxen (PC) & 0.750 & South Korea & 12 \\
\hline$P$ & Desethylatrazine (P) & 0.017 & France & 57 & $\mathrm{PC}$ & Ibuprofen (PC) & 0.773 & South Korea & 12 \\
\hline$P$ & Atrazine $(\mathrm{P})$ & 0.030 & France & 57 & $\mathrm{PC}$ & Sucralose (PC) & 0.818 & South Korea & 12 \\
\hline$P$ & N,N-diéthyl-3-méthylbenzamide (P) & 0.035 & South Korea & 12 & $\mathrm{PC}$ & Iopamidol (PC) & 1.550 & South Korea & 12 \\
\hline $\mathrm{P}$ & Isoproturon $(\mathrm{P})$ & 0.040 & France & 57 & $\mathrm{PC}$ & Iohexol (PC) & 1.770 & South Korea & 12 \\
\hline$P$ & Triclocarban (P) & 0.061 & South Korea & 12 & $\mathrm{PC}$ & Caffeine (PC) & 2,54 & South Korea & 12 \\
\hline
\end{tabular}

How to cite this paper: Gosset, A., Durrieu, C., Orias, F., Bayard, R., Perrodin, Y. (2017) Identification and assessment of ecotoxicological hazards attributable to pollutants in urban wet weather discharges. Environmental Science: Processes \& Impacts DOI: 10.1039/C7EM00159B 
This paper is available at pubs.rsc.org

\begin{tabular}{|c|c|c|c|c|c|c|c|c|c|}
\hline $\mathrm{P}$ & Aminotriazole (P) & 0.460 & France & 57 & $\mathrm{PC}$ & Iopromide (PC) & 2,66 & South Korea & 12 \\
\hline $\mathrm{P}$ & Diuron (P) & 0.470 & France & 57 & РСВ & PCB101 (PCB) & $\mathrm{ND}$ & France & 57 \\
\hline$P$ & AMPA (P) & 1.600 & France; Switzerland & $53 ; 57$ & РСВ & PCB118 (PCB) & ND & France & 57 \\
\hline $\mathrm{P}$ & Glyphosate (P) & 3.4 & France; Switzerland & $53 ; 57$ & РСВ & PCB138 (PCB) & ND & France & 57 \\
\hline PAH & Acenaphthene (PAH) & ND & France & $15 ; 57$ & РСВ & PCB153 (PCB) & $\mathrm{ND}$ & France & 57 \\
\hline PAH & Benzo[a]anthracene (PAH) & ND & France & $15 ; 57$ & РСB & PCB180 (PCB) & $\mathrm{ND}$ & France & 57 \\
\hline PAH & Benzo[b]fluoranthene (PAH) & ND & France & $15 ; 57$ & РСВ & PCB194 (PCB) & ND & France & 57 \\
\hline PAH & Benzo[g,h,i]perylene (PAH) & ND & France & $15 ; 57$ & РСB & РCB28 (PCB) & ND & France & 57 \\
\hline PAH & Benzo[k]fluoranthene (PAH) & $\mathrm{ND}$ & France & $15 ; 57$ & РСВ & PCB52 (PCB) & ND & France & 57 \\
\hline PAH & Chrysene (PAH) & ND & France & $15 ; 57$ & PHT & $\begin{array}{l}\text { Diethylhexyl phthalate } \\
\text { (PHT) }\end{array}$ & $\mathrm{ND}$ & France & 57 \\
\hline PAH & Dibenzo[a,h]anthracene (PAH) & ND & France & $15 ; 57$ & VOC & Ethylbenzene (VOC) & ND & France & 57 \\
\hline PAH & Benzo[a]pyrene (PAH) & 0.005 & France & $15 ; 57$ & VOC & $\begin{array}{l}\text { Tetrachloroethylene } \\
\text { (VOC) }\end{array}$ & ND & France & 57 \\
\hline PAH & Benzo[e]pyrene (PAH) & 0.006 & France & $15 ; 57$ & VOC & Toluene (VOC) & ND & France & 57 \\
\hline PAH & Indeno[1,2,3-cd]pyrene $(\mathrm{PAH})$ & 0.008 & France & $15 ; 57$ & VOC & Trichloroethylene (VOC) & ND & France & 57 \\
\hline PAH & Anthracene (PAH) & 0.009 & France & $15 ; 57$ & VOC & Xylene (VOC) & ND & France & 57 \\
\hline PAH & Acenaphthylene (PAH) & 0.020 & France & $15 ; 57$ & VOC & Hexachlorobenzene (VOC) & 0.002 & $\begin{array}{l}\text { USA (New } \\
\text { York) }\end{array}$ & 56 \\
\hline PAH & Pyrene (PAH) & 0.021 & France & $15 ; 57$ & & & & & \\
\hline PAH & Fluoranthene (PAH) & 0.025 & France & $15 ; 57$ & & & & & \\
\hline PAH & Fluorene (PAH) & 0.027 & France & $15 ; 57$ & & & & & \\
\hline PAH & Phenanthrene (PAH) & 0.085 & France & $15 ; 57$ & & & & & \\
\hline PAH & Naphthalene (PAH) & 0.180 & France & $15 ; 57$ & & & & & \\
\hline
\end{tabular}




\section{This paper is available at pubs.rsc.org}

\subsection{Hazard of pollutants in separate and combined systems}

Using ecotoxicological (PNEC) and chemical ( $\mathrm{MEC}_{\max }$ ) results previously enunciated we were able to calculate a very wide range of HQs in discharges (see Figures 5 and 6). The high dispersion of HQ values calculated could be explained by the heterogeneity of PNEC values observed in Figures 2 to 4 and the heterogeneity of pollutant concentrations due to the high variability of anthropic activities, and thus that of the catchment leached by rain, and the variability of rainfall events (length, height, flow rate, etc.). Due to the relatively different nature and composition of stormwater and CSO, pollutant hazards associated to these two matrices are discussed separately in the following sections.

\section{$\underline{\text { 3.4.1 Hazard quotients in separate systems }}$}

Concerning stormwater samples, only 51 hazard quotients were calculated due to the low number of compounds found in the dissolved part (Figure 5). We found 14 compounds with an HQ lower than 1. The lowest hazard quotient was calculated for Metaldehyde (P) with an HQ of 6.7.10-5. On the contrary, the majority of the micropollutants detected in stormwater samples had an HQ as high as 1 and are therefore potentially hazardous for aquatic ecosystems. Among these 37 compounds, 10 (27.1\%) were pesticides, and 9 PAHs (24.32\%). It is also noteworthy that 4 of the 6 most hazardous compounds (HQ >1000) were pesticides. Among these very hazardous compounds, we also observed the presence of one ALK (4-p-nonylphenol) and one PAH (fluoranthene). The most hazardous compound found was permethrin, with a hazard quotient of 4166.6. The important hazard from pesticides (pyrethroids) linked to high HQ could be explained by the high ecotoxicity of these pesticides rather than by the concentrations found in discharges (around $100 \mathrm{ng} \cdot \mathrm{L}^{-1}$ ). As pointed out before, most of these pesticides (such as bifenthrin, cypermethrin) have a very low PNEC value, lower than $0.1 \mathrm{ng} . \mathrm{L}^{-}$ 1.

\subsubsection{Hazard quotients in combined systems}

Regarding CSO, only $43 \mathrm{HQs}$ were calculated, also due to the low number of molecules measured in dissolved phase in CSO (Figure 6). The HQs obtained were lower than for separate systems.

How to cite this paper: Gosset, A., Durrieu, C., Orias, F., Bayard, R., Perrodin, Y. (2017) Identification and assessment of ecotoxicological hazards attributable to pollutants in urban wet weather discharges. Environmental Science: Processes \& Impacts DOI: 10.1039/C7EM00159B 


\section{This paper is available at pubs.rsc.org}

Indeed, the highest HQ was found for lead, with a value of 68.29. Contrary to separate systems, the highest hazards were due to heavy metals, pesticides and pharmaceutical compounds. Only five families were found in this ranking (HQ >1): PC (3 compounds), M (4), PAH (1), P (5) and ALK (1), and were potentially hazardous for aquatic ecosystems. Results highlighted for heavy metals can be explained by the fact that certain heavy metals in these effluents were in high concentrations (e.g. $2.1 \mu \mathrm{g} . \mathrm{L}^{-1}$ for lead and $1.6 \mu \mathrm{g} . \mathrm{L}^{-1}$ for copper), and not because of their low ecotoxicity, as shown by their relatively high PNEC values. The presence of hazardous PC in CSO can be easily explained by the discharge of large amounts of these often very ecotoxic molecules coming from urban wastewater in stormwater, without any treatment. Comparing hazard quotients from CSO samples to those of separate systems, we can observe that calculated HQs are often lower (e.g. 65.4 in CSO against 950 in stormwater for copper). We can simply explain these results by a dilution of stormwater pollutants by wastewater in CSO.

\subsubsection{The case of pharmaceutical compounds in CSO studies}

With the hazardous pharmaceutical compounds that have been highlighted above, 15 HQs have been calculated only and presented in Figure 6. As urban wastewater is mixed with stormwater in combined sewers, all the pharmaceutical compounds classically consumed in cities could be found in this type of discharge. However, few of them (28) have been taken into account to date in scientific literature. Consequently, our study and all the other ecotoxicological studies dealing with CSO contain a bias attributable to the fact that the potentially considerable hazard represented by these types of molecules is not taken into account. Indeed, as shown by Orias et al. ${ }^{36}$, many of them can have very low PNEC values (e.g. 17ß-estradiol: 8 pg.L $\mathrm{L}^{-1}$, clotrimazole: 0.5 pg.L $\mathrm{L}^{-1}$ ), and/or are widely consumed (e.g. ibuprofen and salicylic acid).

\subsubsection{The case of molecules (e.g., pesticides) forbidden by European legislation}

Some of the pesticides involved in the hazard of UWWDs (found in both stormwater and CSO, such as diuron) were forbidden in 2008 by European legislation. However, several European studies ${ }^{65,66,67}$ have detected the presence of these pesticides (with concentrations reaching $0.64 \mu \mathrm{g} \mathrm{L}^{-1}$ in discharges). This observation can be notably explained by the fact that farmers have stored pesticides How to cite this paper: Gosset, A., Durrieu, C., Orias, F., Bayard, R., Perrodin, Y. (2017) Identification and assessment of ecotoxicological hazards attributable to pollutants in urban wet weather discharges. Environmental Science: Processes \& Impacts DOI: 10.1039/C7EM00159B 


\section{This paper is available at pubs.rsc.org}

345 purchased in the past and that they continue to use. Moreover, some of them linger on in the environment

346 for long periods (due to their high half-life times) and their release can continue due to rainfall events.

347 Consequently, it is true to say that the hazard represented by these pesticides continues to exist even if

348 they are no longer on the market.

How to cite this paper: Gosset, A., Durrieu, C., Orias, F., Bayard, R., Perrodin, Y. (2017) Identification and assessment of ecotoxicological hazards attributable to pollutants in urban wet weather discharges. Environmental Science: Processes \& Impacts DOI: 10.1039/C7EM00159B 


\section{This paper is available at pubs.rsc.org}

\section{Stormwater pollutant hazard quotients}

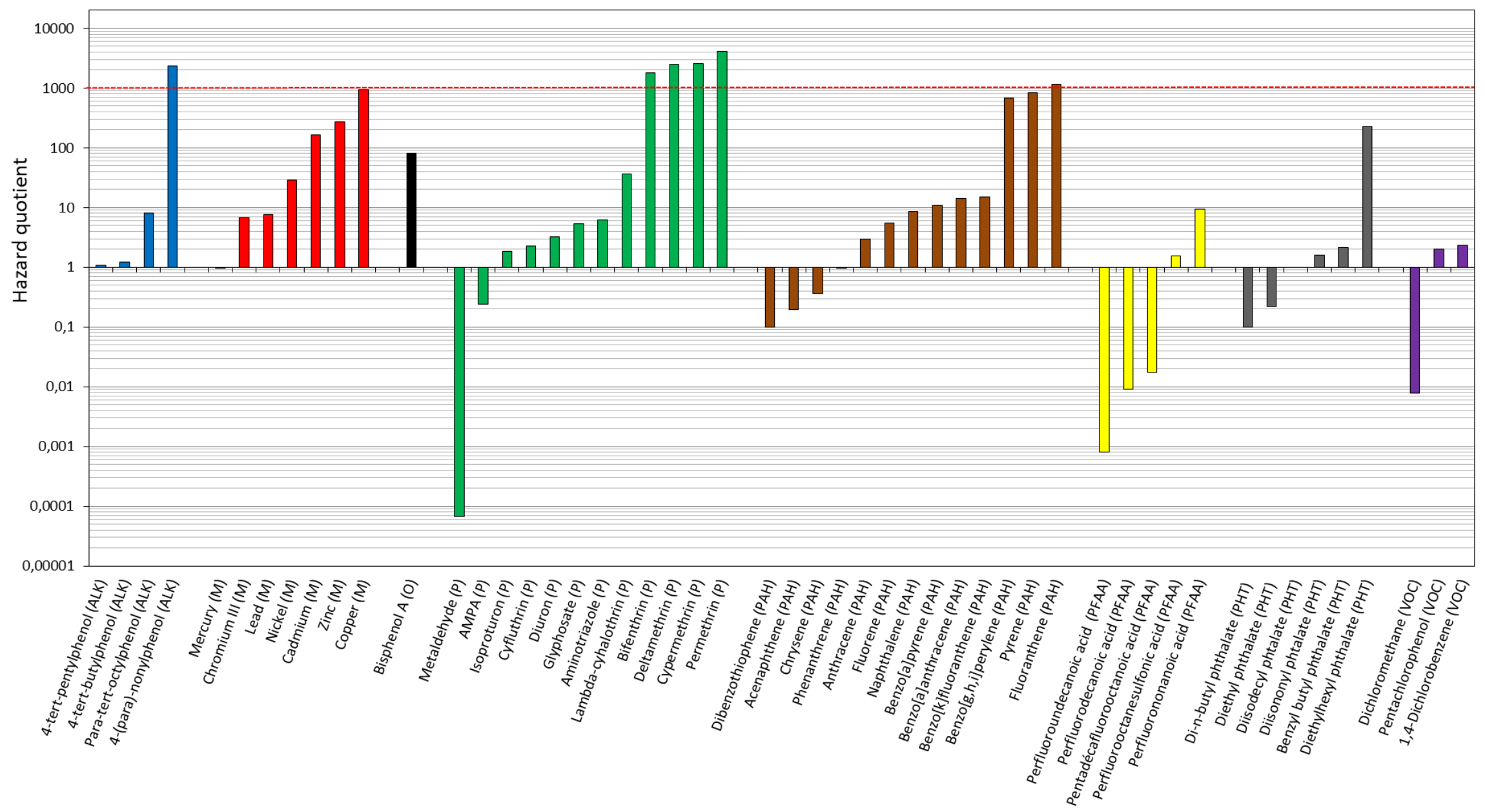

Figure 5: Hazard quotients calculated for micropollutants detected in stormwater samples (the red line delimits the compounds with an HQ up to 1000,

considered very hazardous). Hazard quotients are ranked in ascending order into each separated pollutant family.

How to cite this paper: Gosset, A., Durrieu, C., Orias, F., Bayard, R., Perrodin, Y. (2017) Identification and assessment of ecotoxicological hazards attributable to pollutants in urban wet weather discharges. Environmental Science: Processes \& Impacts DOI: 10.1039/C7EM00159B 


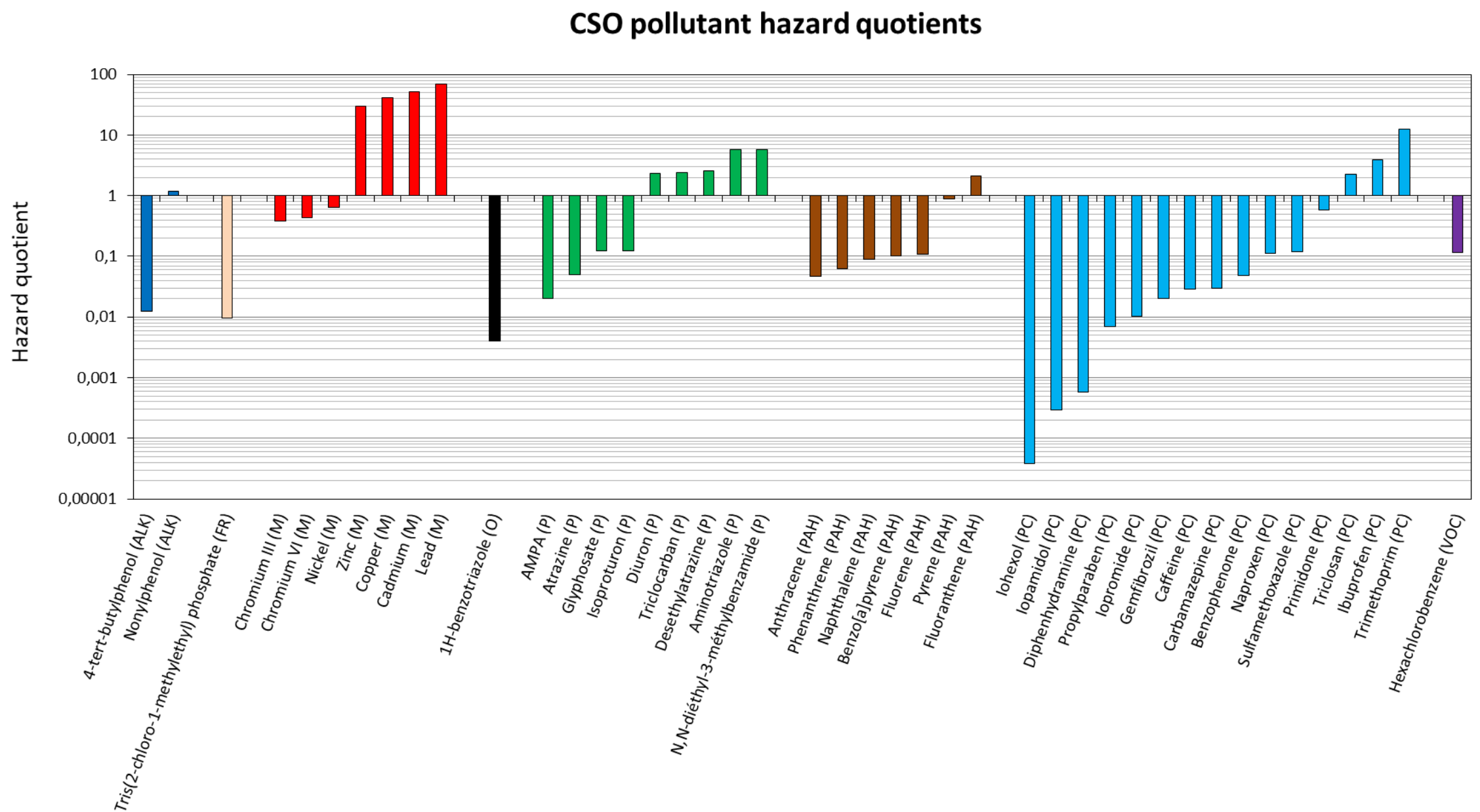

Figure 6: Hazard quotients calculated for micropollutants detected in CSO samples. Hazard quotients are ranked in ascending order into each separated pollutant family. to pollutants in urban wet weather discharges. Environmental Science: Processes \& Impacts DOI: 10.1039/C7EM00159B 


\section{This paper is available at pubs.rsc.org}

\subsection{Discussion of the method and data used}

The method presented in this study proved to be relevant to detect UWWD pollutant hazards. It could be applied for many other matrices, and included in many risk assessment scenarios or chemical prioritization programs. Nevertheless, our method and results are subject to limitations that need to be developed. The first one is the non-consideration of certain parameters, such as the bioconcentration, bioaccumulation $^{30}$ and biomagnification of pollutants in the aquatic food web, which could amplify considerably the hazard of UWWDs pollutants. Indeed, these phenomena have already been demonstrated for many families of pollutants, such as the most hazardous found in UWWDs: heavy metals $^{68}$, pesticides ${ }^{69,70}$ and pharmaceutical compounds..$^{71,72,73,74}$ Other parameters such as $\mathrm{pH}$, temperature, water chemistry ( $\mathrm{pH}$, hardness) can also modify pollutant toxicity. The "mixture" effect, linked to antagonisms of synergisms effects between sought, but also non-potentially thousand molecules not yet identified in UWWDs, are not considered into this study and can modify their hazardousness. Secondly, the use of NOECs for PNEC values construction can be discussed. The authors are aware that ECx values are more accurate than NOEC. ECx values are calculated from the modeling of the dose-response curve, which is itself based on the results of the whole range of concentration, whereas NOEC corresponds only to the identification of the last concentration of the range without effect. However, in the context of this work, it has been chosen to use to the largest set of values available in the literature and in international databases for the two reasons: (1) In the specific context of the PNEC calculation (i.e., a no-effect value for all aquatic organisms), considering the scarcity of existing data in the literature, it seems more useful to consider values for one or several additional species than using accurate values for a limited number of species. In other words, interspecies variability is more important to take into account than intra-species variability, (2) This is the most common approach adopted by international Agencies in charge of the establishment of PNECs for substances, such as INERIS in France, or the European Chemical Bureau in Europe, through the TGD guidelines. Thirdly, our method considers only the maximum concentrations of the different pollutants in UWWDs. Consequently, these values represent only the maximum hazard for each compound. This work could also have been done with median values if sufficient data had been available. This problem How to cite this paper: Gosset, A., Durrieu, C., Orias, F., Bayard, R., Perrodin, Y. (2017) Identification and assessment of ecotoxicological hazards attributable to pollutants in urban wet weather discharges. Environmental Science: Processes \& Impacts DOI: 10.1039/C7EM00159B 


\section{This paper is available at pubs.rsc.org}

381 of limited data collection is at the origin of another limit: most of the chemical studies focusing on

382 dissolved compound concentrations found in stormwater and CSO have dealt with relatively small areas

383 of the world and many of them were published in Europe. Thus data are lacking for some continents, a

384 factor which limits our studies due to differences in, for example, PC consumption, pesticide use (e.g.

385 glyphosate is widely used in South America), sewer systems, etc. Consequently, we can confidently assert that the HQs calculated here are underestimated and would probably increase if more data were available. Fourthly, dissolved part of UWWDs were only considered in this study because the PNEC values were established for dissolved substances and it was impossible to extend them to pollutants whose bioavailability is unknown but present on particles in UWWDs. Indeed, pollutant bioavailability is linked to particle desorption which depends on multiple parameters like $\mathrm{pH}$, molar mass, solubility/lipophily, and the number of carboxyl groups (for organic pollutants). ${ }^{75}$ It is therefore possible that our method underestimated the hazard of these UWWD pollutants for aquatic environments. For example, as in the study of Zgheib et al. ${ }^{4}$ if we had considered the concentration of fluoranthene in the dissolved phase $\left(0.018 \mu \mathrm{g} \mathrm{L}^{-1}\right)$ and the total effluent $\left(0.832 \mu \mathrm{g} \mathrm{L}^{-1}\right)$, assuming that all the pollutants are available, the hazard index of the pollutants would have increased from 1.5 to 69.33 , making it one of the most hazardous compounds for aquatic organisms. This point is illustrated in Figure 7. Similar findings were recorded by Baun et al. ${ }^{30}$ who prioritized 56 organic pollutants in the water phase of stormwater, against 121 in the solid phase. Fifthly, many chemical studies have considered only mineral and organic priority pollutants from the EU Water Framework Directive and other international legislations. However, many emerging compounds currently used present proven ecotoxic effects but have not yet been analyzed. For example, pesticides used massively in Europe like metam-sodium and epoxiconazole present relatively low PNEC values (respectively 0.79 and $0.18 \mu \mathrm{g} \mathrm{L}^{-1}$ ). We can therefore expect high hazard quotients linked to these kinds of molecule. Finally, the presence of high concentrations of bacteria in CSO has already been detected, for example by Passerat et al. (with concentrations up to $10^{7}$ cell/L for E.coli) $\cdot{ }^{76} \mathrm{It}$ is now known that bacteria can transform pollutants and create by-products that are more or less toxic than their parent compounds detected in CSO. These bacteria can therefore influence the composition of CSO and their toxicity with time.

How to cite this paper: Gosset, A., Durrieu, C., Orias, F., Bayard, R., Perrodin, Y. (2017) Identification and assessment of ecotoxicological hazards attributable to pollutants in urban wet weather discharges. Environmental Science: Processes \& Impacts DOI: 10.1039/C7EM00159B 


\section{This paper is available at pubs.rsc.org}

Despite these limits, our method enabled us to highlight new outlooks in terms of prioritization and management of UWWD pollutants. Comparing our results to previous works done with the same purpose, we can observe that considering only intrinsic characteristics (e.g. particle solubility, sorption potential, ecotoxicity) of the pollutants selected, Baun et al. ${ }^{30}$ classified 56 organic compounds as priority pollutants dissolved in water using their RICH (Ranking and Identification of Chemical Hazards) method against 37 in our case (of which 31 are organic). The difference between the two studies is particularly notable for pesticides (10 against 33 pesticides for the method of Baun et al. ${ }^{30}$ ). This difference can be explained by non-detected or low environmental concentrations of many pesticides dissolved in water of our studied discharges. On the contrary, we revealed here 9 hazardous PAH and 3 hazardous PHT instead of respectively 5 and 2 in Baun et al. ${ }^{30}$ An explanation of this discrepancy could be the multiplication of decision filters (sorption/volatilization, technical problems, persistence, bioaccumulation, toxicity) applied by Baun et al. when selecting their compounds, and the lack of data for some pollutants that can lead to the rejection or selection of the priority list, as suggested by these authors. In the case of CSO linked hazard, no studies have investigated a similar prioritization method. Consequently, no comparison can be done.

Looking for the scientific literature, our study enabled us to find new hazardous and priority pollutants. The same observation could be made for the European (Urban Wastewater Directive (UWD 91/271/EEC)) and American (USA EPA) priority pollutant lists. In fact, as stated in Table S2, some pollutant identified here as hazardous such as diuron or fluoranthene are already under the regulation of international instances. On the contrary, some pollutants are absent from these lists. For example, some heavy metals (e.g. copper and zinc in UWD list), pesticides as glyphosate or pyrethrinoids in stormwater, phthalates (e.g. diisononyl phthalate) and pharmaceuticals such as ibuprofen or trimethoprim in CSO absent in UWD and EPA priority lists, are revealed to be at the origin of an important hazard for aquatic receiving ecosystems, and thus should be so the object of reinforced controls and could be proposed to be added to these lists.

How to cite this paper: Gosset, A., Durrieu, C., Orias, F., Bayard, R., Perrodin, Y. (2017) Identification and assessment of ecotoxicological hazards attributable to pollutants in urban wet weather discharges. Environmental Science: Processes \& Impacts DOI: 10.1039/C7EM00159B 


\section{This paper is available at pubs.rsc.org}

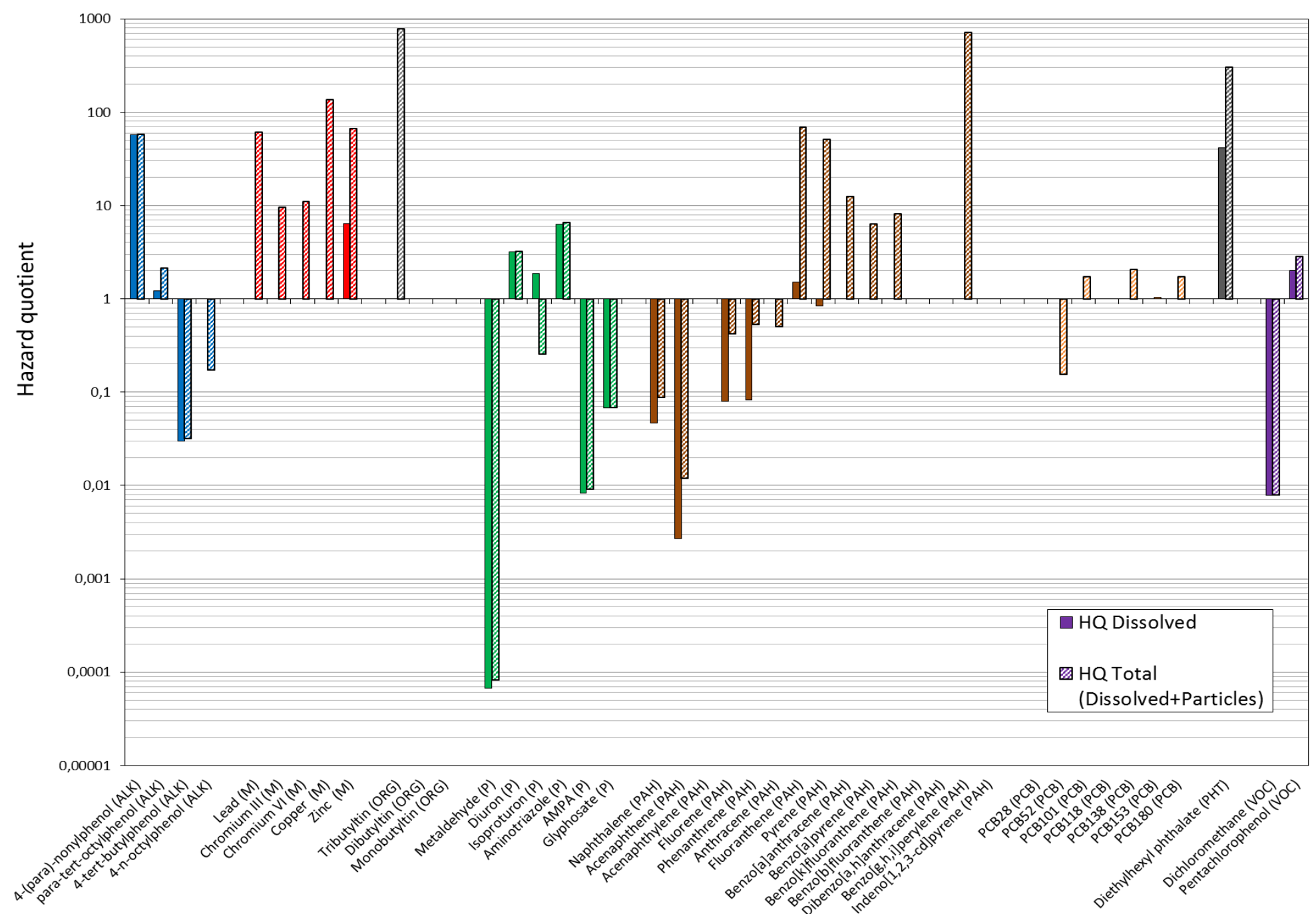




\section{This paper is available at pubs.rsc.org}

435

\section{Conclusion and perspectives}

The aim of our study was to develop and apply a methodology to determine and calculate hazard quotients associated with the different pollutants sought and found in UWWDs. A wide range of pollutants was sought in the latter, with highly variable ecotoxicity and concentrations. Although many families of pollutants (PC, PAH, VOC, ALK, M, P, O, PFAA and PHT) play a role in the UWWD hazards identified, we emphasize that they are comprise of a large number of pesticides and heavy metals (e.g. pyrethrinoïds, copper and cadmium). The hazards of the pollutants identified in this study have different origins: (i) the presence of high concentrations of certain highly ecotoxic compounds (e.g. Pyrene in stormwater), (ii) the presence of high concentrations of slightly ecotoxic compounds (e.g. heavy metals in CSO), (iii) the presence of weak concentrations of certain highly ecotoxic compounds (e.g. pesticides like Bifenthrin and Permethrin in stormwater samples), (iv) the presence of intermediate concentrations of compounds with intermediate ecotoxicity (e.g. alkylphenols in UWWDs). We showed that the hazard linked to CSO was lower than that linked to stormwater from separate systems. However, these results should be considered with caution because only a few pharmaceutical compounds (just 28 compounds) were sought in CSO, compared to the huge range of drugs consumed by human beings. Moreover, no ecotoxicity data were available for 13 of the compounds (3 PC, 6 PAH, 2 ALK, 1 ORG, 1 PFAA) detected in UWWDs, and therefore no PNEC or HQ values could be calculated. Consequently, it will be necessary in further studies to obtain more ecotoxicity data on these compounds. Moreover, current hazard and risk assessment methods must be improved to take into account pollutant bioconcentration/accumulation, which can represent a serious risk for higher trophic levels.

To conclude, this work enabled us to identify the potential hazard of UWWDs and the pollutants involved. We now know which pollutants require an in-depth monitoring. We have also identified different points which require in-depth studies. In particular, it is now necessary to better assess the hazard of certain pollutants bound to the particulate phase. Lastly, this work should help managers to identify the largest and most hazardous pollution sources in order to treat them in priority.

How to cite this paper: Gosset, A., Durrieu, C., Orias, F., Bayard, R., Perrodin, Y. (2017) Identification and assessment of ecotoxicological hazards attributable to pollutants in urban wet weather discharges. Environmental Science: Processes \& Impacts DOI: 10.1039/C7EM00159B 


\section{This paper is available at pubs.rsc.org}

462

463

464

465

466

467

468

469

470

471

472

473

474

475

476

477

478

479

480

481

482

483

484

485

486

487

488

489

490

491

$\underline{\text { Acknowledgements }}$

This work was funded by the French Minister of Ecology and Sustainable Development, the French

Minister of Higher Education and Research and the University of Lyon (Faculté des Sciences et Technologies). The authors thank Dr. Laurent Lassabatère for helpful discussions about the article. They also thank the anonymous referees for their constructive reviews.

$\underline{\text { References }}$

1 M. Kafi, J. Gasperi, R. Moilleron, M. C. Gromaire and G. Chebbo, Water Research, 2008, 42, 539-549.

2H.-M. Hwang and G. D. Foster, Environmental Pollution, 2006, 140, 416-426.

3S. Terzakis, M. S. Fountoulakis, I. Georgaki, D. Albantakis, I. Sabathianakis, A. D.

Karathanasis, N. Kalogerakis and T. Manios, Chemosphere, 2008, 72, 141-149.

4S. Zgheib, R. Moilleron, M. Saad and G. Chebbo, Water Research, 2011, 45, 913-925.

5 Y. Kalmykova, K. Björklund, A.-M. Strömvall and L. Blom, Water Research, 2013, 47, $1317-1328$.

6 K. Björklund, A. P. Cousins, A.-M. Strömvall and P.-A. Malmqvist, Science of The Total Environment, 2009, 407, 4665-4672.

7 M. Cladière, J. Gasperi, C. Lorgeoux, C. Bonhomme, V. Rocher and B. Tassin, Environ Sci Pollut Res, 2013, 20, 2973-2983.

8J. Gasperi, S. Garnaud, V. Rocher and R. Moilleron, Science of The Total Environment, 2008, 407, 263-272.

9 E. Gooré Bi, F. Monette, J. Gasperi and Y. Perrodin, Environmental Science and Pollution Research, 2015, 22, 4460-4474.

10 C. Becouze-Lareure, A. Dembélé, M. Coquery, C. Cren-Olivé, B. Barillon and J.-L.

Bertrand-Krajewski, Urban Water Journal, 2016, 13, 600-617.

11 S. Deffontis, A. Breton, C. Vialle, M. Montréjaud-Vignoles, C. Vignoles and C.

Sablayrolles, Science of The Total Environment, 2013, 452, 394-403.

12 J. Ryu, J. Oh, S. A. Snyder and Y. Yoon, Environ Monit Assess, 2014, 186, 3239-3251.

13 X. Peng, Y. Yu, C. Tang, J. Tan, Q. Huang and Z. Wang, Science of The Total

Environment, 2008, 397, 158-166.

14 J.-Y. Pailler, A. Krein, L. Pfister, L. Hoffmann and C. Guignard, Science of The Total

How to cite this paper: Gosset, A., Durrieu, C., Orias, F., Bayard, R., Perrodin, Y. (2017) Identification and assessment of ecotoxicological hazards attributable to pollutants in urban wet weather discharges. Environmental Science: Processes \& Impacts DOI: 10.1039/C7EM00159B 


\section{This paper is available at pubs.rsc.org}

492

493

494

495

496

497

498

499

500

501

502

503

504

505

506

507

508

509

510

511

512

513

514

515

516

517

518

519

520

521

522

523

524

Environment, 2009, 407, 4736-4743.

15 R. Angerville, Y. Perrodin, C. Bazin and E. Emmanuel, International Journal of

Environmental Research and Public Health, 2013, 10, 2670-2687.

16 L. Wu, Y. Jiang, L. Zhang, L. Chen and H. Zhang, Environmental Science and Pollution Research, 2014, 21, 2663-2676.

17 R.-H. Chen, F.-P. Li, H.-P. Zhang, Y. Jiang, L.-C. Mao, L.-L. Wu and L. Chen, Science of The Total Environment, 2016, 553, 519-523.

18 M. Lafont, L. Grapentine, Q. Rochfort, J. Marsalek, G. Tixier and P. Breil, Water Science and Technology, 2007, 56, 13-20.

19 C. Parent-Raoult and J.-C. Boisson, Revue des sciences de l'eau / Journal of Water Science, 2007, 20, 229-239.

20 K. A. Johnson, A. D. Steinman, W. D. Keiper and C. R. Ruetz, Journal of the North American Benthological Society, 2011, 30, 710-727.

21 C. M. Kinsella and T. P. Crowe, Marine Environmental Research, 2015, 105, 20-29.

22 Y. Ferro, M. Perullini, M. Jobbagy, S. Bilmes and C. Durrieu, Sensors, 2012, 12, 1687916891.

23 A. Gosset, Y. Ferro and C. Durrieu, Water Research, 2016, 89, 330-354.

24 A. Wigh, A. Devaux, V. Brosselin, A. Gonzalez-Ospina, B. Domenjoud, S. Aït-Aïssa, N.

Creusot, A. Gosset, C. Bazin and S. Bony, Environmental Science and Pollution Research, 2016, 23, 3008-3017.

25 F. Orias and Y. Perrodin, Chemosphere, 2014, 115, 31-39.

26 M. Gros, M. Petrović, A. Ginebreda and D. Barceló, Environment International, 2010, 36, $15-26$.

27 A. Tsaboula, E.-N. Papadakis, Z. Vryzas, A. Kotopoulou, K. Kintzikoglou and E.

Papadopoulou-Mourkidou, Environment International, 2016, 91, 78-93.

28 W. Li, Y. Shi, L. Gao, J. Liu and Y. Cai, Chemosphere, 2012, 89, 1307-1315.

29 E. Eriksson, A. Baun, P. S. Mikkelsen and A. Ledin, Water Science and Technology, 2005, 51, 47-55.

30 A. Baun, E. Eriksson, A. Ledin and P. S. Mikkelsen, Science of The Total Environment, 2006, 370, 29-38.

31 E. Eriksson, A. Baun, L. Scholes, A. Ledin, S. Ahlman, M. Revitt, C. Noutsopoulos and P.

S. Mikkelsen, Science of The Total Environment, 2007, 383, 41-51.

32 E. Eriksson, A. Baun, P. S. Mikkelsen and A. Ledin, Desalination, 2007, 215, 187-197.

How to cite this paper: Gosset, A., Durrieu, C., Orias, F., Bayard, R., Perrodin, Y. (2017) Identification and assessment of ecotoxicological hazards attributable to pollutants in urban wet weather discharges. Environmental Science: Processes \& Impacts DOI: 10.1039/C7EM00159B 


\section{This paper is available at pubs.rsc.org}

525

526

527

528

529

530

531

532

533

534

535

536

537

538

539

540

541

542

543

544

545

546

547

548

549

550

551

552

553

554

555

556

557

558

33 AGRITOX database on plant protection substances. http://www.agritox.anses.fr/ (December 2016)

34 INERIS database, http://www.ineris.fr/rapports-d\%C3\%A9tude/toxicologie-etenvironnement/fiches-de-donn\%C3\%A9es-toxicologiques-et-environnementales, (December 2016)

35 EPA ECOTOX database, http://www.epa.gov/ecotox/, (December 2016)

36 F. Orias and Y. Perrodin, Science of The Total Environment, 2013, 454-455, 250-276.

37 TGD, Technical Guidance Document in Support of Commission Directive 93/67/EEC on Risk Assessment for New Notified Substances and Commission Regulation (EC), 2003.

38 H. Sanderson, D. J. Johnson, C. J. Wilson, R. A. Brain and K. R. Solomon, Toxicology Letters, 2003, 144, 383-395.

39 R. J. Kieber, J. D. Willey and S. D. Zvalaren, Environ. Sci. Technol., 2002, 36, 5321-5327.

40 D. P. Weston, R. W. Holmes and M. J. Lydy, Environmental Pollution, 2009, 157, 287-294.

41 C. S. Eckley and B. Branfireun, Science of The Total Environment, 2008, 403, 164-177.

42 S.-K. Kim and K. Kannan, Environ. Sci. Technol., 2007, 41, 8328-8334.

43 Y. Zushi and S. Masunaga, Environmental Toxicology and Chemistry, 2009, 28, 691-700.

44 V. T. Nguyen, M. Reinhard and G. Y.-H. Karina, Chemosphere, 2011, 82, 1277-1285.

45 F. Xiao, M. F. Simcik and J. S. Gulliver, Water Research, 2012, 46, 6601-6608.

46 M. Murakami, H. Shinohara and H. Takada, Chemosphere, 2009, 74, 487-493.

47 R. Pitt, R. Field, M. Lalor, M. Brown, Water Environment Research, 67, 260-275.

48 B. Ngabe, T.F. Bidleman and G.I. Scott, Science of The Total Environment, 2000, 255, 1-9.

49 L. Herngren, A. Goonetilleke, G. A. Ayoko and M. M. M. Mostert, Environmental Pollution, 2010, 158, 2848-2856.

50 M. N. Chong, J. Sidhu, R. Aryal, J. Tang, W. Gernjak, B. Escher and S. Toze, Environmental Monitoring and Assessment, 2013, 185, 6645-6652.

51 Q. Zhang, X. Wang, P. Hou, W. Wan, Y. Ren, Z. Ouyang and L. Yang, Environmental Monitoring and Assessment, 2013, 185, 9763-9775.

52 M. Huber, A. Welker and B. Helmreich, Science of The Total Environment, 2016, 541, 895-919.

53 I. Hanke, I. Wittmer, S. Bischofberger, C. Stamm and H. Singer, Chemosphere, 2010, 81, $422-429$.

54 G. Imfeld, M. Lefrancq, E. Maillard and S. Payraudeau, Chemosphere, 2013, 90, 1333-1339.

55 M. Kayhanian, C. Suverkropp, A. Ruby and K. Tsay, Journal of Environmental Management, 2007, 85, 279-295.

56 E. Pratt, K. Irvine, S. Marshall, B. G. Loganathan, S. Kumar and H. C. Sikka, Journal of

Water Management Modeling, 1995.

How to cite this paper: Gosset, A., Durrieu, C., Orias, F., Bayard, R., Perrodin, Y. (2017) Identification and assessment of ecotoxicological hazards attributable to pollutants in urban wet weather discharges. Environmental Science: Processes \& Impacts DOI: 10.1039/C7EM00159B 


\section{This paper is available at pubs.rsc.org}

559

560

561

562

563

564

565

566

567

568

569

570

571

572

573

574

575

576

577

578

579

580

581

582

583

584

585

586

587

588

589

590

591

57 J. Gasperi, S. Zgheib, M. Cladière, V. Rocher, R. Moilleron and G. Chebbo, Water Research, 2012, 46, 6693-6703.

58 J. Gasperi, M. C. Gromaire, M. Kafi, R. Moilleron and G. Chebbo, Water Research, 2010, 44, 5875-5886.

59 R. Mulliss, D. M. Revitt and R. B. E. Shutes, Water Science and Technology, 1997, 36, 195199.

60 M. Karvelas, A. Katsoyiannis and C. Samara, Chemosphere, 2003, 53, 1201-1210.

61 N. Bolong, A. F. Ismail, M. R. Salim and T. Matsuura, Desalination, 2009, 239, 229-246.

62 T. Deblonde, C. Cossu-Leguille and P. Hartemann, International Journal of Hygiene and Environmental Health, 2011, 214, 442-448.63 Luo et al., 2014

64 M. A. Launay, U. Dittmer and H. Steinmetz, Water Research, 2016, 104, 82-92.

65 Stachel, J.-U. Holthuis, W. Schulz, W. Seitz, W. H. Weber, K.-T. Tegge and I. Dobner, in

Xenobiotics in the Urban Water Cycle, eds. D. Fatta-Kassinos, K. Bester and K.

Kümmerer, Springer Netherlands, 2010, pp. 445-461.

66 Zgheib, R. Moilleron and G. Chebbo, Water Research, 2012, 46, 6683-6692.

67 M. P. Ensminger, R. Budd, K. C. Kelley and K. S. Goh, Environ Monit Assess, 2013, 185, $3697-3710$.

68 Y. Tao, Z. Yuan, H. Xiaona and M. Wei, Ecotoxicology and Environmental Safety, 2012, 81, 55-64.

69 S. Ruangsomboon and L. Wongrat, Aquatic Toxicology, 2006, 78, 15-20.

70 T. Katagi, in Review of Environmental Contamination and Toxicology Volume 204, ed. D.

M. Whitacre, Springer New York, New York, NY, 2010, vol. 204, pp. 1-132.

71 Coogan et al., 2007

72 F. Orias, L. Simon, G. Mialdea, A. Clair, V. Brosselin and Y. Perrodin, Ecotoxicology and Environmental Safety, 2015, 120, 457-462.

73 F. Orias, L. Simon and Y. Perrodin, Chemosphere, 2015, 122, 251-256.

74 A. Zenker, M. R. Cicero, F. Prestinaci, P. Bottoni and M. Carere, Journal of

Environmental Management, 2014, 133, 378-387.

75 D. Lachassagne, M. Casellas, A. Gonzalez-Ospina and C. Dagot, TSM, 2016, 31-43.

76 J. Passerat, N. K. Ouattara, J.-M. Mouchel, V. Rocher and P. Servais, Water Research, 2011, 45, 893-903.

How to cite this paper: Gosset, A., Durrieu, C., Orias, F., Bayard, R., Perrodin, Y. (2017) Identification and assessment of ecotoxicological hazards attributable to pollutants in urban wet weather discharges. Environmental Science: Processes \& Impacts DOI: 10.1039/C7EM00159B 


\section{This paper is available at pubs.rsc.org}

593 Table S1: Extrapolation Factor assessment criteria derivate from Orias and Perrodin ${ }^{36}$ and TDG $^{37}$.

\begin{tabular}{|l|c|}
\hline Available data & EF (Extrapolation Factor) \\
\hline $\begin{array}{l}\text { At least one short-term L(E)C50 from each } \\
\text { of three trophic levels of the base-set } \\
\text { (e.g. algae, daphnids and fishes) }\end{array}$ & 1000 \\
\hline $\begin{array}{l}\text { One long term chronic data } \\
\text { (e.g. invertebrate or fish) }\end{array}$ & 100 \\
\hline $\begin{array}{l}\text { Two long term chronic data from species representing } \\
\text { two trophic levels (e.g.algae and/or daphnids and/or fishes) }\end{array}$ & 50 \\
\hline $\begin{array}{l}\text { Two long term chronic data from species representing } \\
\text { three trophic levels (e.g. algae, daphnids and fishes) }\end{array}$ & 10 \\
\hline $\begin{array}{l}\text { Statistics method (e.g. species sensitivity distribution (SSD)), } \\
\text { field data or model ecosystem }\end{array}$ & $5-1$ (Reviewed case by case) \\
\hline
\end{tabular}

594

595

596

597

598

599

600

601

602

603

604

605

606

607

608

609

610

611

612

613

614

How to cite this paper: Gosset, A., Durrieu, C., Orias, F., Bayard, R., Perrodin, Y. (2017) Identification and assessment of ecotoxicological hazards attributable to pollutants in urban wet weather discharges. Environmental Science: Processes \& Impacts DOI: 10.1039/C7EM00159B 


\begin{tabular}{|c|c|c|c|c|}
\hline \multirow[b]{2}{*}{ Pollutant } & \multicolumn{2}{|c|}{ Identified as hazardous } & \multicolumn{2}{|c|}{ Identification as a priority subtance } \\
\hline & $\begin{array}{c}\text { In } \\
\text { stormwater }\end{array}$ & $\begin{array}{l}\text { In combined } \\
\text { sewer } \\
\text { overflows }\end{array}$ & $\begin{array}{c}\text { In European Water Framework } \\
\text { Directive (WFD, 2000/60/EC) } \\
\text { pollutant priority list }\end{array}$ & $\begin{array}{l}\text { In Environmental Protection } \\
\text { Agency pollutant priority list }\end{array}$ \\
\hline 4-(para)-nonylphenol (ALK) & Yes & No & Yes & No \\
\hline 4-tert-pentylphenol (ALK) & Yes & No & Yes & No \\
\hline 4-tert-butylphenol (ALK) & Yes & No & Yes & No \\
\hline Nonylphenol (ALK) & No & Yes & Yes & No \\
\hline Para-tert-octylphenol (ALK) & Yes & No & Yes & No \\
\hline Cadmium $(\mathrm{M})$ & Yes & Yes & Yes & Yes \\
\hline Chromium III (M) & Yes & No & No & Yes \\
\hline Copper (M) & Yes & Yes & No & Yes \\
\hline Lead $(\mathrm{M})$ & Yes & Yes & Yes & Yes \\
\hline Nickel (M) & Yes & No & Yes & Yes \\
\hline $\operatorname{Zinc}(\mathrm{M})$ & Yes & Yes & No & Yes \\
\hline Bisphenol A (O) & Yes & No & No & No \\
\hline Aminotriazole $(\mathrm{P})$ & Yes & Yes & No & No \\
\hline Bifenthrin $(\mathrm{P})$ & Yes & No & No & No \\
\hline Cyfluthrin (P) & Yes & No & No & No \\
\hline Cypermethrin (P) & Yes & No & Yes & No \\
\hline Deltamethrin (P) & Yes & No & No & No \\
\hline Desethylatrazine $(\mathrm{P})$ & Yes & Yes & No & No \\
\hline Diuron $(\mathrm{P})$ & Yes & Yes & Yes & No \\
\hline Glyphosate (P) & Yes & No & No & No \\
\hline Isoproturon $(\mathrm{P})$ & Yes & No & Yes & No \\
\hline Lambda-cyhalothrin (P) & Yes & No & No & No \\
\hline $\begin{array}{l}\text { N,N-diéthyl-3- } \\
\text { méthylbenzamide (P) }\end{array}$ & No & Yes & No & No \\
\hline Permethrin $(\mathrm{P})$ & Yes & No & No & No \\
\hline Triclocarban (P) & No & Yes & No & No \\
\hline Anthracene (PAH) & Yes & No & Yes & Yes \\
\hline Benzo[a]anthracene (PAH) & Yes & No & Yes & Yes \\
\hline Benzo[k]fluoranthene (PAH) & Yes & No & Yes & Yes \\
\hline Benzo[g,h,i]perylene (PAH) & Yes & No & Yes & Yes \\
\hline Benzo[a]pyrene (PAH) & Yes & No & Yes & Yes \\
\hline Fluoranthene (PAH) & Yes & Yes & Yes & Yes \\
\hline Fluorene (PAH) & Yes & No & Yes & Yes \\
\hline Naphthalene (PAH) & Yes & No & Yes & Yes \\
\hline Pyrene (PAH) & Yes & No & Yes & Yes \\
\hline Ibuprofen (PC) & No & Yes & No & No \\
\hline Triclosan (PC) & No & Yes & No & No \\
\hline Trimethoprim (PC) & No & Yes & No & No \\
\hline $\begin{array}{l}\text { Perfluorooctanesulfonic acid } \\
\text { (PFAA) }\end{array}$ & Yes & No & Yes & No \\
\hline Perfluorononanoic acid (PFAA) & Yes & No & Yes & No \\
\hline Benzyl butyl phthalate (PHT) & Yes & No & No & Yes \\
\hline Diethylhexyl phthalate (PHT) & Yes & No & Yes & Yes \\
\hline Diisononyl phthalate (PHT) & Yes & No & No & No \\
\hline 1,4-Dichlorobenzene (VOC) & Yes & No & No & Yes \\
\hline
\end{tabular}

How to cite this paper: Gosset, A., Durrieu, C., Orias, F., Bayard, R., Perrodin, Y. (2017) Identification and assessment of ecotoxicological hazards attributable to pollutants in urban wet weather discharges. Environmental Science: Processes \& Impacts DOI: 10.1039/C7EM00159B 
This paper is available at pubs.rsc.org classification of these compounds as priority pollutants or not by European Water Framework Directive and In Environmental Protection Agency. 